\title{
Comparative genomics reveals multistep pathogenesis of E2A-PBX1 acute lymphoblastic leukemia
}

\author{
Jesús Duque-Afonso, ${ }^{1}$ Jue Feng, ${ }^{1,2}$ Florian Scherer, ${ }^{3}$ Chiou-Hong Lin, ${ }^{1}$ Stephen H.K. Wong, ${ }^{1}$ Zhong Wang, ${ }^{1}$ Masayuki Iwasaki, ${ }^{1}$ \\ and Michael L. Cleary ${ }^{1}$ \\ 'Department of Pathology, Stanford University School of Medicine, Stanford, California, USA. ²Department of Microbiology and Immunology, Columbia University Medical Center, New York, New York, USA. \\ ${ }^{3}$ Divisions of Oncology and Hematology, Department of Medicine, Stanford University School of Medicine, Stanford, California, USA.
}

\begin{abstract}
Acute lymphoblastic leukemia (ALL) is the most common childhood cancer; however, its genetic diversity limits investigation into the molecular pathogenesis of disease and development of therapeutic strategies. Here, we engineered mice that conditionally express the E2A-PBX1 fusion oncogene, which results from chromosomal translocation $t(1 ; 19)$ and is present in $5 \%$ to $7 \%$ of pediatric ALL cases. The incidence of leukemia in these mice varied from $5 \%$ to $50 \%$, dependent on the Credriving promoter ( $C d 19, M b 1$, or $M x 1)$ used to induce E2A-PBX1 expression. Two distinct but highly similar subtypes of B cell precursor ALLs that differed by their pre-B cell receptor (pre-BCR) status were induced and displayed maturation arrest at the pro-B/large pre-B II stages of differentiation, similar to human E2A-PBX1 ALL. Somatic activation of E2A-PBX1 in B cell progenitors enhanced self-renewal and led to acquisition of multiple secondary genomic aberrations, including prominent spontaneous loss of Pax5. In preleukemic mice, conditional Pax5 deletion cooperated with E2A-PBX1 to expand progenitor B cell subpopulations, increasing penetrance and shortening leukemia latency. Recurrent secondary activating mutations were detected in key signaling pathways, most notably JAK/STAT, that leukemia cells require for proliferation. These data support conditional E2A-PBX1 mice as a model of human ALL and suggest targeting pre-BCR signaling and JAK kinases as potential therapeutic strategies.
\end{abstract}

\section{Introduction}

Leukemias are malignant disorders of blood-forming cells that primarily result from acquired aberrations of the genome. The consistent association of specific chromosomal rearrangements observed cytogenetically in distinct subsets of leukemia $(1,2)$ prompted the initial hypothesis that leukemias may result from subtype-specific genetic abnormalities (3). Subsequent extensive molecular and genomic studies led to a more refined 2-mutation model for leukemia pathogenesis, in which one genetic lesion activates a kinase-driven signaling pathway to confer a proliferative advantage, and a cooperating second mutation corrupts a transcription factor to block the differentiation of normal progenitor cells (4). More recent genomic studies using nextgeneration sequencing technologies have shown that leukemias are genetically more complex and diverse than previously appreciated. Genomic studies of human acute lymphoblastic leukemia (ALL), in particular, have suggested a 3-step model of leukemia pathogenesis (5), which postulates that an initiating genetic lesion such as E2A-PBX1 (also known as TCF3-PBX1), ETV6-RUNX1 (TEL-AML1), or $M L L$ fusions confers self-renewal properties to hematopoietic stem cells (HSCs) or lymphoid progenitors. A second lesion, such as $P A X 5, E B F 1$, or IKFZ1, normally affecting essential

\section{Related Commentary: p. 3427}

Authorship note: Jesús Duque-Afonso and Jue Feng contributed equally to this work. Conflict of interest: The authors have declared that no conflict of interest exists. Submitted: January 26, 2015; Accepted: June 18, 2015.

Reference information: J Clin Invest. 2015;125(9):3667-3680. doi:10.1172/JCI81158. transcription factors for B cell development, causes differentiation block at the progenitor B cell level $(6,7)$. A third class of cooperating mutations accumulate and are needed to fully transform leukemia cells, affecting pathways such as cell cycle $(C D K N 2 A / B$, TP53), cytokine receptors and associated kinases (JAK kinases, CRLF2, IL7R), RAS signaling (NRAS, KRAS, PTPN11), or several other transcription factors or epigenetic regulators $(8,9)$. However, this recently articulated 3-step model of leukemogenesis, which is based on elegant genomic studies, has not been prospectively tested in vivo or validated in an experimental system.

We addressed this issue in the current study by modeling ALL caused by the $\mathrm{t}(1 ; 19)$ chromosomal translocation, which is present in $5 \%$ to $7 \%$ of pediatric ALLs. This genomic rearrangement fuses the transcription factor E2A (TCF3) with the homeobox gene $P B X 1(10,11)$ to serve as the initiating lesion in a phenotypically and genetically distinctive subtype of ALL. We demonstrate that activation of E2A-PBX1 in B cell progenitors induces 2 different subtypes of leukemia based on the presence of pre-BCR, enhances self-renewal, and leads to acquisition of multiple genomic aberrations including prominent loss of PAX5 and activation of JAK/STAT signaling. Our findings credential the efficacy of targeting pre-BCR signaling and JAK kinases as therapeutic strategies in ALL.

\section{Results}

Conditional E2A-PBX1 activation and E2A haploinsufficiency in the hematopoietic compartment of mice. To investigate the cellular roles of E2A-PBX1 in leukemogenesis, we developed mouse strains that conditionally activate and express the E2A-PBX1 fusion gene in 
B cell progenitors. Somatic activation of the oncogene was accomplished by Cre recombinase expressed under the control of specific B lineage promoters $C d 19$ or $M b 1$ (Ig $\alpha, C D 79 a)$ or in hematopoietic stem cells using the $M x 1$ promoter (Figure $1 \mathrm{~A}$ ). To monitor E2A-PBX1 recombination and expression at the single-cell level by flow cytometry, the GFP gene preceded by an internal ribosomal entry site (IRES) element was engineered into the targeted allele. GFP expression was detected mainly in $\mathrm{CD}_{1}{ }^{+} \mathrm{B}$ cells $(\sim 90 \%)$ and less frequently in $\mathrm{T}$ cell subsets $(3 \%)$ and mature myeloid $\mathrm{CD} 11 \mathrm{~b}^{+}$cells $(\sim 5 \%)$ in the peripheral blood of recombined mice (data not shown).

Western blot analysis confirmed the expression of E2A-PBX1 protein in sorted $\mathrm{GFP}^{+} \mathrm{BM}$ progenitor B cells ( $\mathrm{Lin}^{-} \mathrm{CD} 19^{+} \mathrm{CD} 43^{+}$) in 3-month-old healthy preleukemic mice, whereas WT E2A protein levels were reduced by $50 \%$ compared with normal B cell progenitors (Figure 1B). These results demonstrate specific, conditional expression of E2A-PBX1 in the hematopoietic compartment and provide a model in which E2A-PBX1 expression is activated concomitant with induction of $E 2 A$ haploinsufficiency to recapitulate the oncogenetics associated with $t(1 ; 19)$ chromosomal translocations in human ALL.

Conditional E2A-PBX1 Tg mice consistently develop acute leukemia. Mice that conditionally expressed E2A-PBX1 developed leukemia with latencies and penetrance that varied for the different Cre lines. Leukemia incidence at 12 months ranged from $7 \%$ for the $\operatorname{Tg}(E 2 A-P B X 1) C d 19-C r e$ line to $53 \%$ for the $\operatorname{Tg}(E 2 A-P B X 1)$ $M b 1-C r e$ line and 59\% for the $\operatorname{Tg}(E 2 A-P B X 1) M x 1$-Cre line (Figure $1 C)$, suggesting that the earlier Cre recombinase is expressed during $\mathrm{B}$ cell development, the higher the penetrance of E2A-PBX1 leukemias. GFP expression was detected in BM cells of moribund mice, confirming expression of the $E 2 A-P B X 1 \mathrm{Tg}$ in leukemia cells (Figure 1D). Leukemic mice presented with splenomegaly (Figure $1 \mathrm{~F}$ ), lymphadenopathy (Supplemental Figure 1A; supplemental material available online with this article; doi:10.1172/ JCI81158DS1), hepatomegaly (Supplemental Figure 1B), anemia, thrombocytopenia, and leukocytosis (Figure 1G). Leukemia cells (Figure 1E) were present in the BM, spleen, and lymph nodes and infiltrated multiple organs (Supplemental Figure 1C), including the CNS (Supplemental Figure 1D). BM leukemia cells were transplantable into sublethally irradiated recipient mice, inducing secondary leukemia after 3 to 4 weeks (Supplemental Figure $1 \mathrm{E})$. Pathologic and immunophenotypic characterization showed that $94 \%$ of 52 leukemias had a B cell precursor phenotype characterized by expression of CD19, CD43, and GFP, whereas 2 were of myeloid lineage and 1 was of $\mathrm{T}$ lineage. Thus, conditional activation of $E 2 A-P B X 1$ in the hematopoietic compartment predominantly induces B cell precursor leukemia.

E2A-PBX1 leukemic blasts are arrested at the pro-/pre-B stage of $B$ cell precursor differentiation. The B cell precursor leukemias comprised 2 subtypes distinguished by their pre-B cell receptor status (pre-BCR ${ }^{+}$vs. pre-BCR ${ }^{-}$). Both types displayed similar immunophenotypes (surface CD19 ${ }^{+}, \mathrm{CD} 43^{+}, \mathrm{B} 220 / \mathrm{CD} 45^{+}, \mathrm{CD} 25^{+}, \mathrm{Bp}-1^{+}$, $\mathrm{CD} 24 / \mathrm{HAS}^{+}, \mathrm{CD} 127 / \mathrm{IL}-7 \mathrm{ra}^{+}, \mathrm{CD} 117^{+}$, and intracellular VPREB, $\mathrm{CD} 79 \mathrm{a}^{+}$, and $\left.\mathrm{TdT}^{+}\right)$, however pre-BCR ${ }^{+}$leukemias were distinguished by cytoplasmic $\mu$ and surface VPREB expression, which are respective components of the pre-BCR (Figure 2A).

Quantification of progenitor B cell subpopulations defined by flow cytometry showed a 10-fold expansion of the B-C' fraction (as defined by $\mathrm{Lin}^{-} \mathrm{CD} 19^{+} \mathrm{CD} 43^{+}$expression) in total BM cells from E2A-PBX1 leukemic mice, compared with those from WT and healthy preleukemic mice. A marked reduction of fraction D-E-F cells (Lin ${ }^{-} \mathrm{CD} 19^{+} \mathrm{CD} 43^{-}$) was observed in the $\mathrm{BM}$ of leukemic and healthy preleukemic mice, suggesting that E2A-PBX1 imposes a differentiation block in $\mathrm{B}$ cell development (Figure 2B). The immunophenotypes correspond to the pro-B/large pre-B II stages (Basel nomenclature) or B-C' stages (Hardy nomenclature) of B cell differentiation (12), which are dependent on the presence or absence of pre-BCR (Figure 2C).

Functional differences between pre- $B C R^{+}$and pre-BCR $E 2 A-$ $P B X 1$ leukemias. Clonal rearrangements of Ig heavy chain VDJ segments were detected in at least $50 \%$ of mouse E2A-PBX1 leukemias by genomic PCR (data not shown), but productive ORFs were present in only $12.5 \%$ of leukemias (Figure $3 \mathrm{~A}$ ), which correlated with high levels of cytoplasmic $\mu$ and surface VPREB expression $\left(\right.$ pre-BCR ${ }^{+}$). Bcl6 expression levels, which are a key feature of active pre-BCR $(13,14)$, were 10 -fold higher in pre-BCR ${ }^{+}$compared with pre-BCR- leukemias (Figure 3B). However, high $\mathrm{Bcl} 6$ expression was observed in 1 pre- $\mathrm{BCR}^{-}$leukemia that contained a nonsense mutation of the $\mathrm{Bcl} 6$ corepressor (Bcor) gene detected in whole-exome sequencing (WES). Pre-BCR ${ }^{+}$leukemias responded to the stimulation of pre-BCR by anti-IgM in the presence of $\mathrm{H}_{2} \mathrm{O}_{2}$ (15), with an increase in phosphorylated PLC $\gamma 2$ (Figure 3C).

Pre- $\mathrm{BCR}^{+}$leukemias were more sensitive in vitro (Figure 3D) to the inhibitory effects of dasatinib, an SRC-family kinase inhibitor that has recently been shown to have promising preclinical efficacy in the treatment of pre-BCR ${ }^{+} \mathrm{ALL}$, including $\mathrm{E} 2 \mathrm{~A}-\mathrm{PBX} 1^{+}$leukemias (13). The leukemia with a concomitant Bcor mutation and high Bcl6 expression levels was resistant to dasatinib, comparable to that seen in pre-BCR- leukemias (Figure 3D). In vivo dasatinib treatment of pre- $\mathrm{BCR}^{+}$leukemia in a secondary transplantation assay led to prolonged disease-free survival compared with that of vehicle-treated mice (Figure 3E). Forced expression of functional $\mu$ heavy chain converted pre-BCR ${ }^{-}$leukemias to pre-BCR ${ }^{+}$, as shown by increased surface VPREB, and conferred dasatinib sensitivity in vitro, indicating that heavy chain status is a critical determinant of the functional differences in the 2 subtypes of E2APBX1 $1^{+}$ALLs (Figure 3, F-H).

E2A-PBX1 perturbs early $B$ progenitor cell differentiation. To determine the potential effects of conditional E2A-PBX1 expression on B cell differentiation, peripheral blood cells were quantified in preleukemic mice at different time points using flow cytometry. A substantially lower percentage of B cells was observed at all time points over a 30 -week period in healthy preleukemic $\operatorname{Tg}(E 2 A-P B X 1) C d 19$-Cre mice compared with that observed in control Cd19-Cre mice (Figure 4A), consistent with the significant decrease in immature and recirculating B cells in the $\mathrm{BM}$ (Figure $2 \mathrm{~B}$ ). Similar reductions were observed in preleukemic $\operatorname{Tg}(E 2 A-P B X 1)$ Mb1-Cre and $\operatorname{Tg}(E 2 A-P B X 1)$ Mx1-Cre mice (data not shown). To further assess $B$ cell differentiation, progenitor B cells were prospectively isolated by FACS and placed in methylcellulose culture supplemented with IL-7, SCF, and FLT3 ligand (FLT3L). Progenitor B cells of $\operatorname{Tg}(E 2 A-P B X 1)$ Cd19-Cre mice proliferated extensively (Figure $4 \mathrm{~B}$ ) and were severely compromised in their ability to differentiate into $\mathrm{CD} 43^{-}$cells compared with progenitor B cells isolated from control mice, which 
A

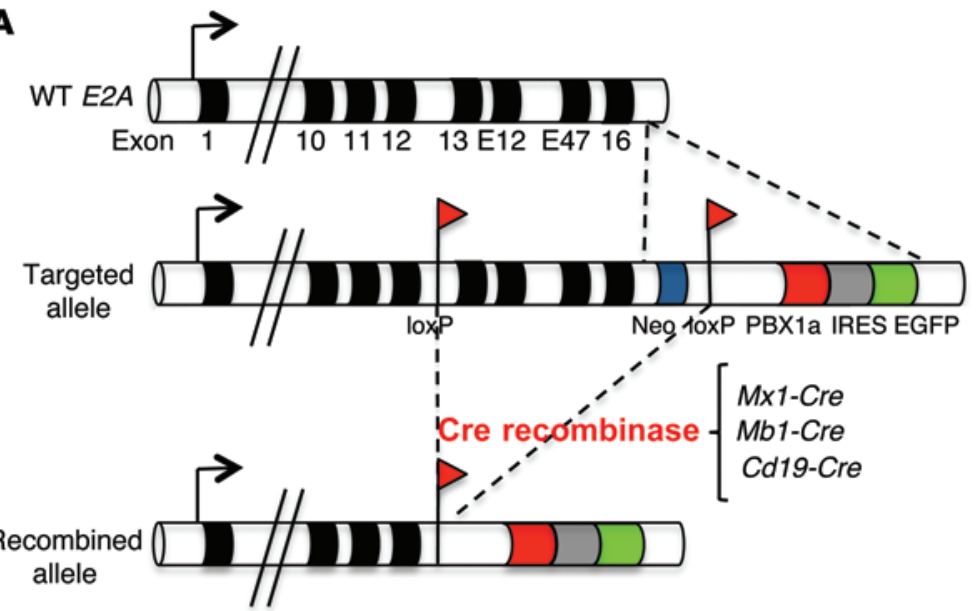

D

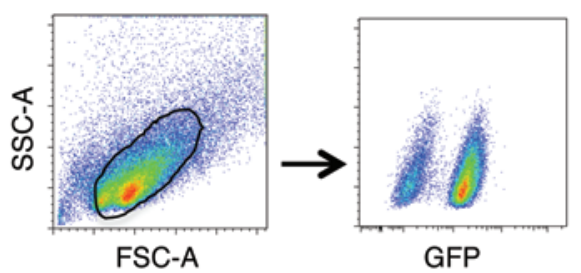

E

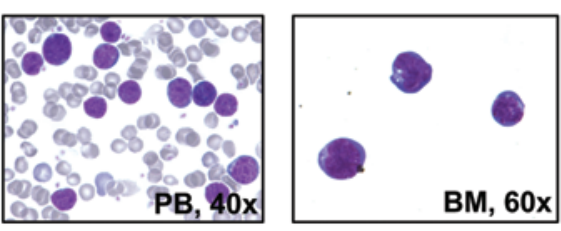

B

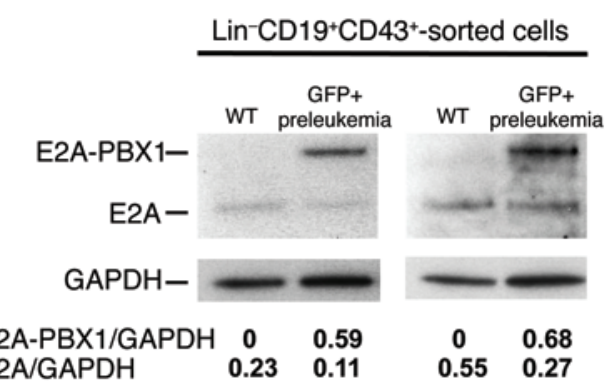

$\mathbf{F}$
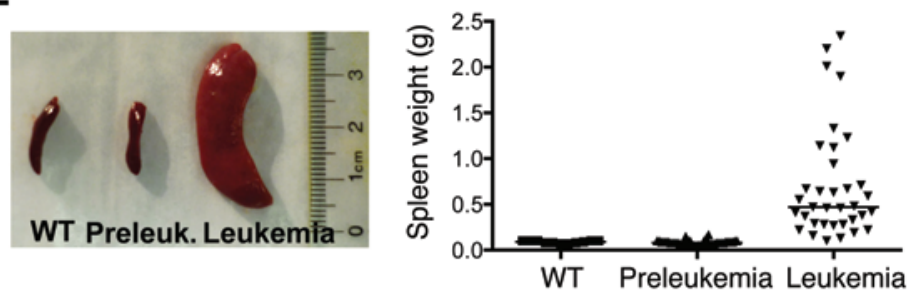

G

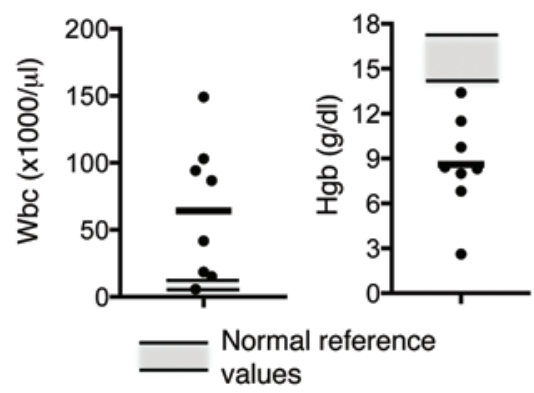

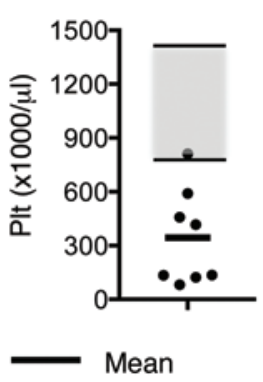

Figure 1. Conditional E2A-PBX1 Tg mice consistently develop leukemia. (A) Schematic representation of WT, targeted, and recombined E2A alleles. Cre-mediated recombination results in deletion of $3^{\prime}$ E2A exons (13, E12, E47, and 16) and the PGK neocassette (neo), fusing in-frame the human PBX1a cDNA linked with EGFP by an IRES element. Cre-recombinase was expressed from the B cell-specific promoter Cd19 or Mb1 (CD79a, Ig $\alpha$ ), or in HSCs from the Mx1 promoter. (B) Representative Western blots show E2A and E2A-PBX1 protein levels in sorted progenitor B cells from WT (Lin-CD19+CD43+) and healthy preleukemic (Lin-CD19+CD43+GFP+) Tg(E2A-PBX1) Cd19-Cre mice. The ratio of E2A/GAPDH and E2A-PBX1/GAPDH levels (shown below) was determined by densitometry. (C) Kaplan-Meier plots show disease-free survival of conditional E2A-PBX1 mice crossed with the Cre-recombinase lines Cd19 ( $n=153)$, Mb1 ( $n=74)$, and $M \times 1(n=44)$. The incidence of leukemia at 12 months is shown on the right. (D) Flow cytometric plots show GFP expression in BM cells from a leukemic mouse. (E) May-Grünwald Giemsa staining of peripheral blood smear (PB) and BM cytospin (BM) show leukemic blast morphology. (F) Spleens are shown for representative WT, preleukemic, and leukemic mice (left panel). Graph shows spleen weights from WT ( $n=11)$, healthy E2A-PBX1 preleukemic $(n=42)$, and leukemic $(n=35)$ mice (horizontal bars denote the mean) (right panel). (C) Hematologic findings at leukemia presentation $(n=8)$. Gray shadows represent normal reference values; horizontal bars denote the mean for the analyzed mice. Hgb, hemoglobin; Plt, platelets, wbc, white blood cells.

mostly differentiated during the 10-day culture (Figure 4C). Thus, E2A-PBX1 impairs the differentiation and enhances the proliferation of progenitor $B$ cells in vitro and in vivo.

E2A-PBX1 enhances B cellprogenitor self-renewal. To assess B cell progenitor turnover, we investigated whether the progenitor B cell population expressing E2A-PBX1 could be reconstituted following irradiation. Young $\operatorname{Tg}(E 2 A-P B X 1) C d 19$-Cre and WT mice were irradiated sublethally to deplete the endogenous B cell populations and assessed by flow cytometry for B cell progenitor recovery. WT mice showed normal regeneration of $B$ cell progenitors with appropriate proportions of all B cell subsets (data not shown). Regeneration also occurred in preleukemic $\operatorname{Tg}(E 2 A-P B X 1)$ Cd19-Cre mice but was distinguished by a dramatically expanded population of $\mathrm{B}^{2} 2 \mathrm{O}^{\text {lo }}$ progenitor $\mathrm{B}$ cells that were almost exclusively $\mathrm{GFP}^{+}$, indicating that E2A-PBX1-expressing progenitor B cells have a competitive advantage ( $\sim 90$-fold) in vivo compared with 
A
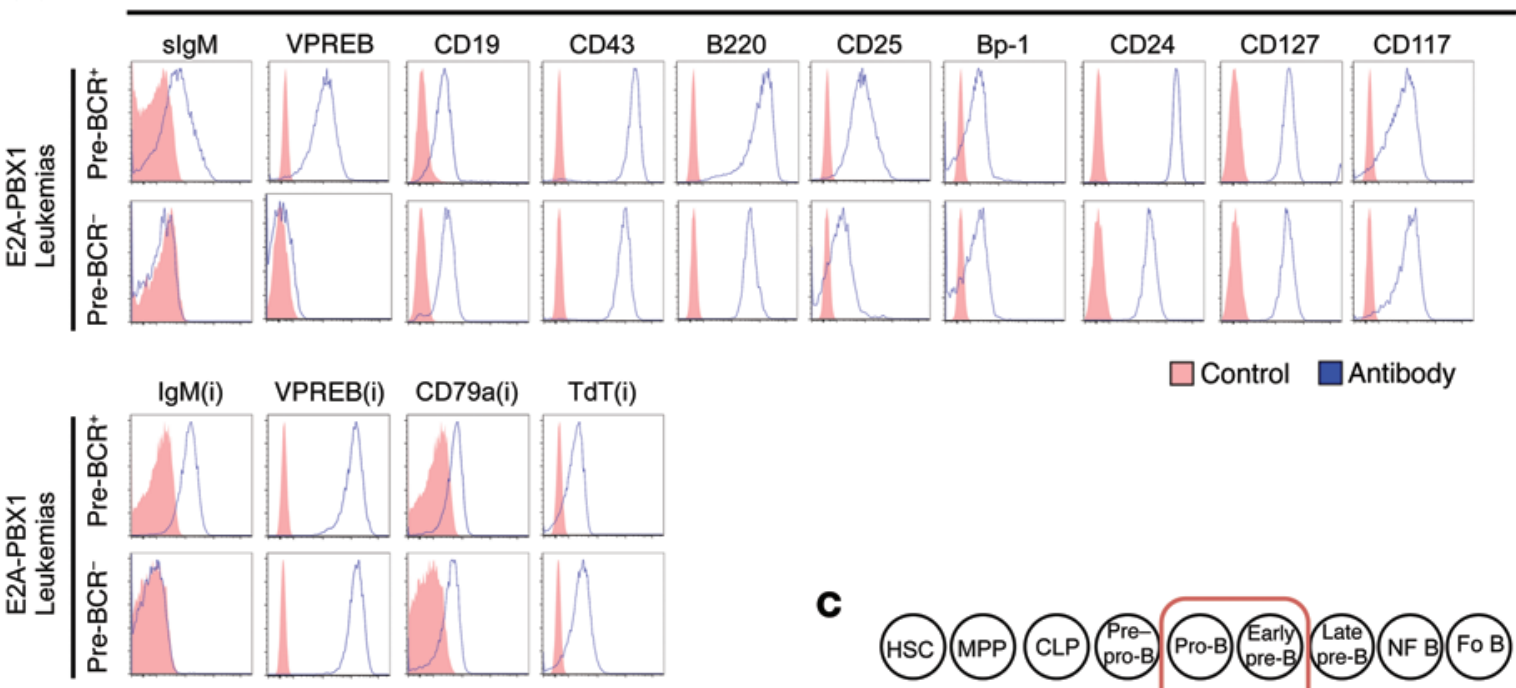

C

B
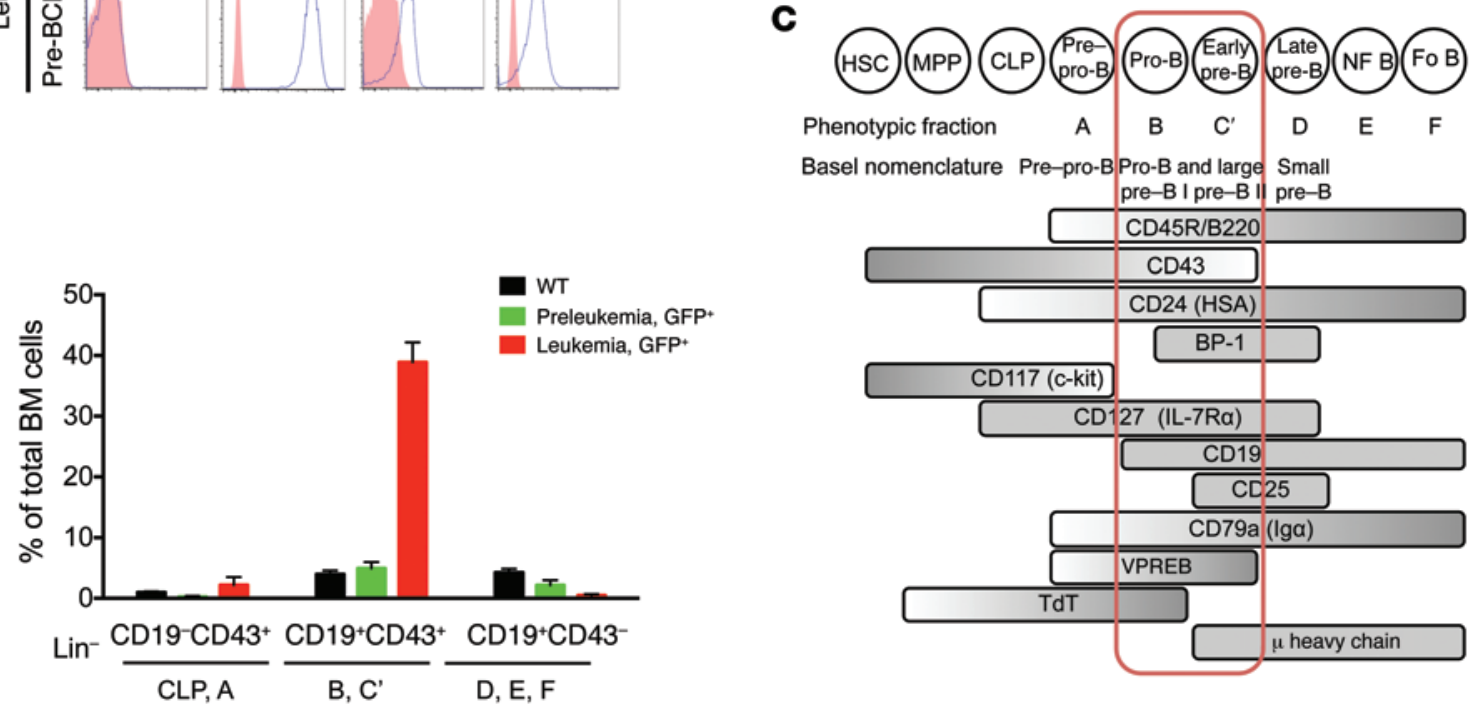

Figure 2. E2A-PBX1 leukemic blasts display B cell precursor immunophenotypes. (A) Histograms show the immunophenotypic profiles for the indicated surface and intracellular (i) markers in GFP+ leukemic blasts of representative pre-BCR+ and pre-BCR- leukemias from 8 analyzed leukemias. Higher levels of cytoplasmic $\mu$ heavy chain (cylgM) and surface VPREB correlated with productive VDJ rearrangement. Red shadow represents control unstained cells for cell-surface markers and isotype controls for intracellular staining. Blue line represents antigen expression. (B) Quantification of progenitor B cell subpopulations in the BM at 3 months of age for WT $(n=7)$, healthy E2A-PBX1 preleukemic $(n=8)$, and leukemic $(n=20)$ mice using an Ab cocktail for lineage markers (CD3, CD4, CD8, Gr1, CD11b, NK1-1, Ter119), CD19, and CD43. (C) Schematic representation of cell surface and intracellular markers expressed in leukemic GFP+ blasts, which correlate with the B-C' differentiation stage (pro-B, pre-B I, large pre-B II) of B cell development (modified from ref. 12). CLP, common lymphoid progenitors; Fo B, follicular B cells; HSA, heat-stable Ag, MPP, multipotential progenitors; NF B, newly formed B cells .

non-E2A-PBX1-expressing (GFP-) progenitor B cells in the same host (Figure 4D). Of note, the B220 bright progenitor B cell population was composed of $50 \% \mathrm{GFP}^{+}$cells (data not shown).

The foregoing studies raised the possibility that E2A-PBX1expressing B lineage progenitors may have stem-like self-renewal properties. To assess this, $\mathrm{CD} 19^{+}$cells harvested from WT or preleukemic $\operatorname{Tg}(E 2 A-P B X 1)$ Cd19-Cre donor mice were transplanted into sublethally irradiated NOD.Cg-Prkdc ${ }^{\text {scid }} \mathrm{I} / 2 \mathrm{rg}^{\text {tmIWil }} / \mathrm{SzJ}$ (NSG) recipient mice, which lack endogenous B cell populations. Analysis of BM 6 weeks after transplantation showed that B cell subsets including pro-B, pre-B, and immature $\mathrm{B}$ cells were reconstituted in recipients of $\mathrm{CD}_{1} 9^{+} \mathrm{BM}$ cells from WT donor mice, consistent with the long-lived growth potential of B cell progenitors (data not shown). B lineage reconstitution was also observed in recipients of $\mathrm{CD} 19^{+} \mathrm{BM}$ cells from preleukemic $\mathrm{Tg}(E 2 A-P B X 1)$
Cd19-Cre donors; however, the vast majority of progenitor $\mathrm{B}$ cells $\left(\mathrm{Lin}^{-} \mathrm{CD} 19^{+} \mathrm{CD}^{2} 3^{+}\right)$in the recipient $\mathrm{BM}$ were $\mathrm{GFP}^{+}$. The difference in the proportion of $\mathrm{GFP}^{+}$versus $\mathrm{GFP}^{-} \mathrm{CD}^{+} 9^{+}$cells was approximately 50 -fold after reconstitution, even though they were present in equal proportions in the donors prior to transplantation (Figure $4 \mathrm{E}$ ). $\mathrm{GFP}^{+}$progenitor B cells were capable of repopulating the BM in secondary and tertiary recipients, while retaining a limited capacity to differentiate into immature B cells (data not shown). These data indicate a cell-autonomous enhancement of self-renewal induced by E2A-PBX1 specifically at the pro-B/pre-B cell stage of maturation, consistent with their markedly impaired differentiation and enhanced proliferation in vitro compared with WT and Cd19-Cre mice.

The differences in leukemia incidence and latency (Figure 1C) raised the possibility that preleukemic E2A-PBX1 cells devel- 
A

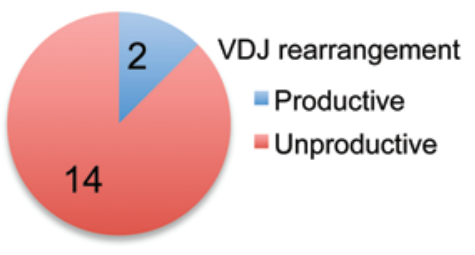

B

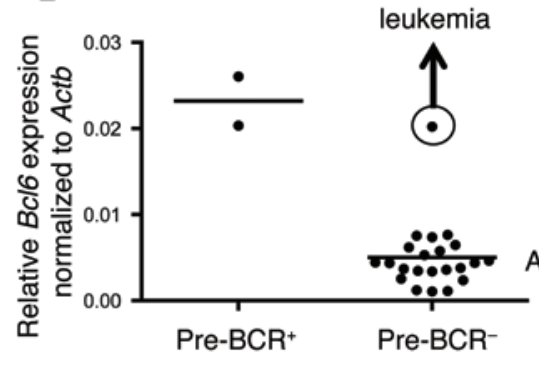

C

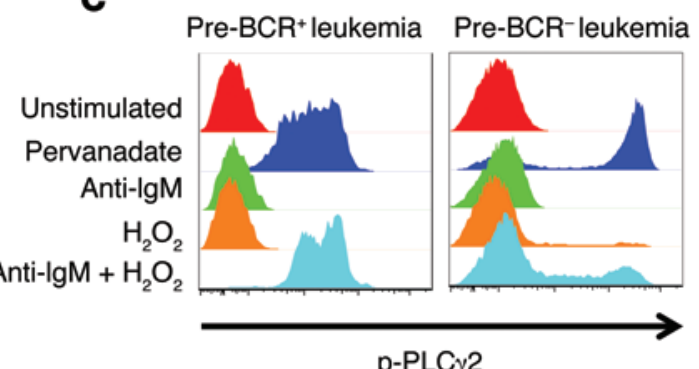

D

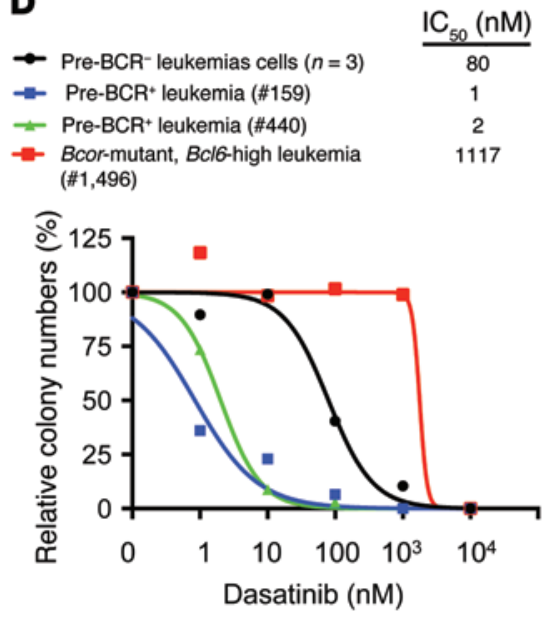

E

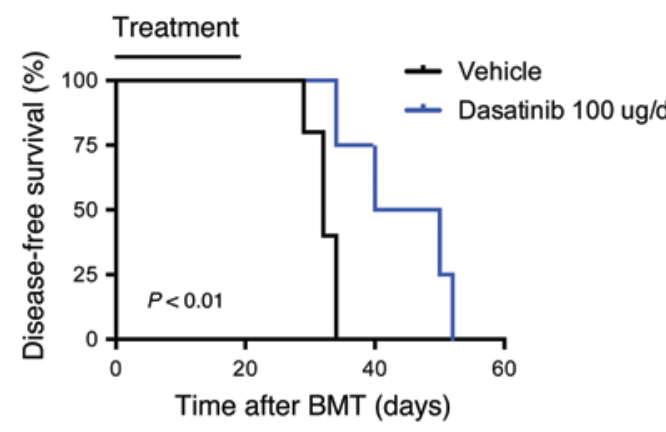

$\mathbf{F}$

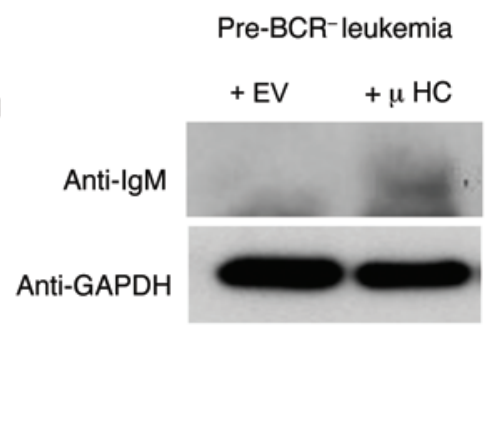

G

Pre-BCR-leukemia
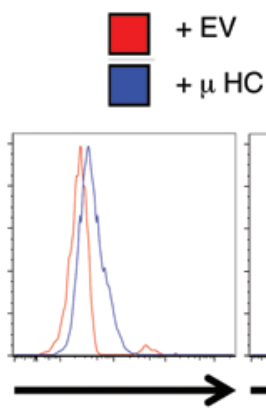

cylgM

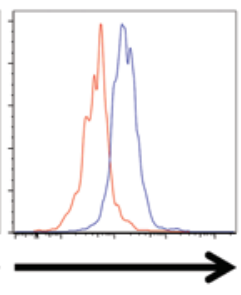

SVPREB
H

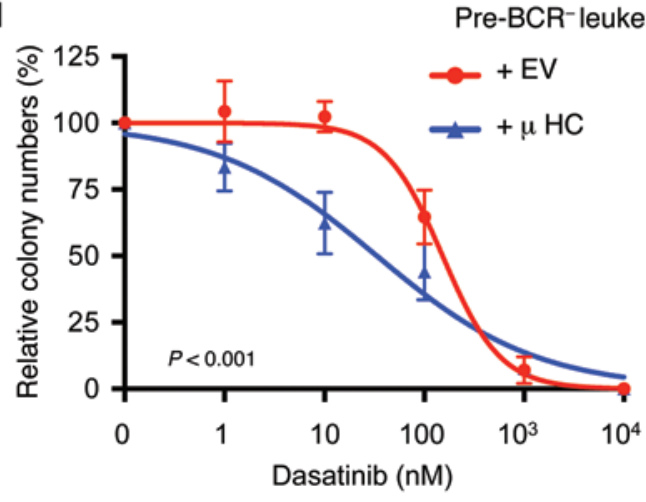

Figure 3. Expression of pre-BCR components in E2A-PBX1 leukemic blasts determines a phenotype very similar to that of human pre-BCR ${ }^{+}$leukemias. (A) Pie chart represents the proportion of analyzed leukemias $(n=16)$ with a productive or unproductive VDJ rearrangement. (B) Bcl6 expression was assessed by qPCR of sorted Lin-CD19+CD43+ cells from BM leukemias $(n=24)$. Leukemias were compared depending on their pre-BCR status. Horizontal bars denote the mean. (C) PLC $\gamma 2$ phosphorylation was analyzed by phospho-flow after pre-BCR stimulation using polyclonal anti-lgM and $\mathrm{H}_{2} \mathrm{O}_{2}$ for 30 minutes (15). Pre-BCR+ and pre-BCR- results of 2 analyzed leukemias from each group are shown. Pervanadate was used as a positive control. (D) BM leukemic cells were cultured in the presence of increasing concentrations of dasatinib. Colonies were enumerated after 7 days. (E) E2A-PBX1 leukemia cells from pre-BCR+ leukemia were transplanted into sublethally irradiated secondary recipients. Mice were treated with vehicle $(n=5)$ or dasatinib $(100 \mu \mathrm{g}$ i.p. daily, $n=4$ ) for 20 days. Statistical analysis was performed by log-rank test. BMT, BM transplantation. (F) Pre-BCR- leukemias were transduced retrovirally to ectopically express empty vector $(\mathrm{EV})$ or functional $\mu$ heavy chain $(\mu \mathrm{HC})$. Results of a representative of 2 transduced leukemias are shown. Western blot shows expression of $\mu$ heavy chain and GAPDH as a loading control. (G) Cytoplasmic lgM (cylgM) and surface VPREB (sVPREB) were assessed by flow cytometry. (H) Transduced pre-BCR- leukemias were cultured at increasing concentrations of dasatinib. Colonies were enumerated after 5 days, and results are expressed as the mean \pm SEM of 3 independent experiments. Statistical analysis was performed using the $F$ test.

oped different properties, depending on the timing of Cre-driver gene activation. In support of this, the frequency of $\mathrm{GFP}^{+} \mathrm{B}$ cell progenitors $\left(\mathrm{Lin}^{-} \mathrm{CD} 19^{+} \mathrm{CD} 43^{+}\right)$in preleukemic $\mathrm{Tg}(E 2 A-P B X 1)$ $M b 1$-Cre mice was significantly higher, as were their GFP mean fluorescence intensity (MFI) values, compared with preleuke- mic $\operatorname{Tg}(E 2 A-P B X 1)$ Cd19-Cre mice (Figure $4 \mathrm{~F})$. Hence, sorted preleukemic $\operatorname{Tg}(E 2 A-P B X 1) M b 1-C r e$ B cell progenitors showed a higher proliferative capacity compared with that of preleukemic $\operatorname{Tg}(E 2 A-P B X 1) C d 19$-Cre cells (Figure $4 G$ ), suggesting that earlier activation of E2A-PBX1 was more oncogenic. 

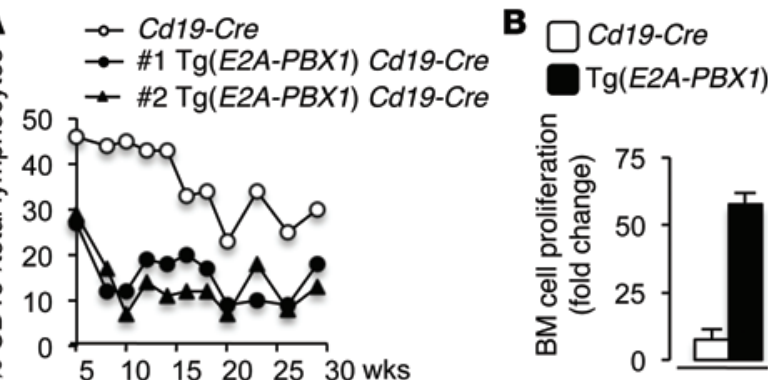

D

\section{E $\operatorname{Tg}(E 2 A-P B X 1)$ Cd19-Cre}
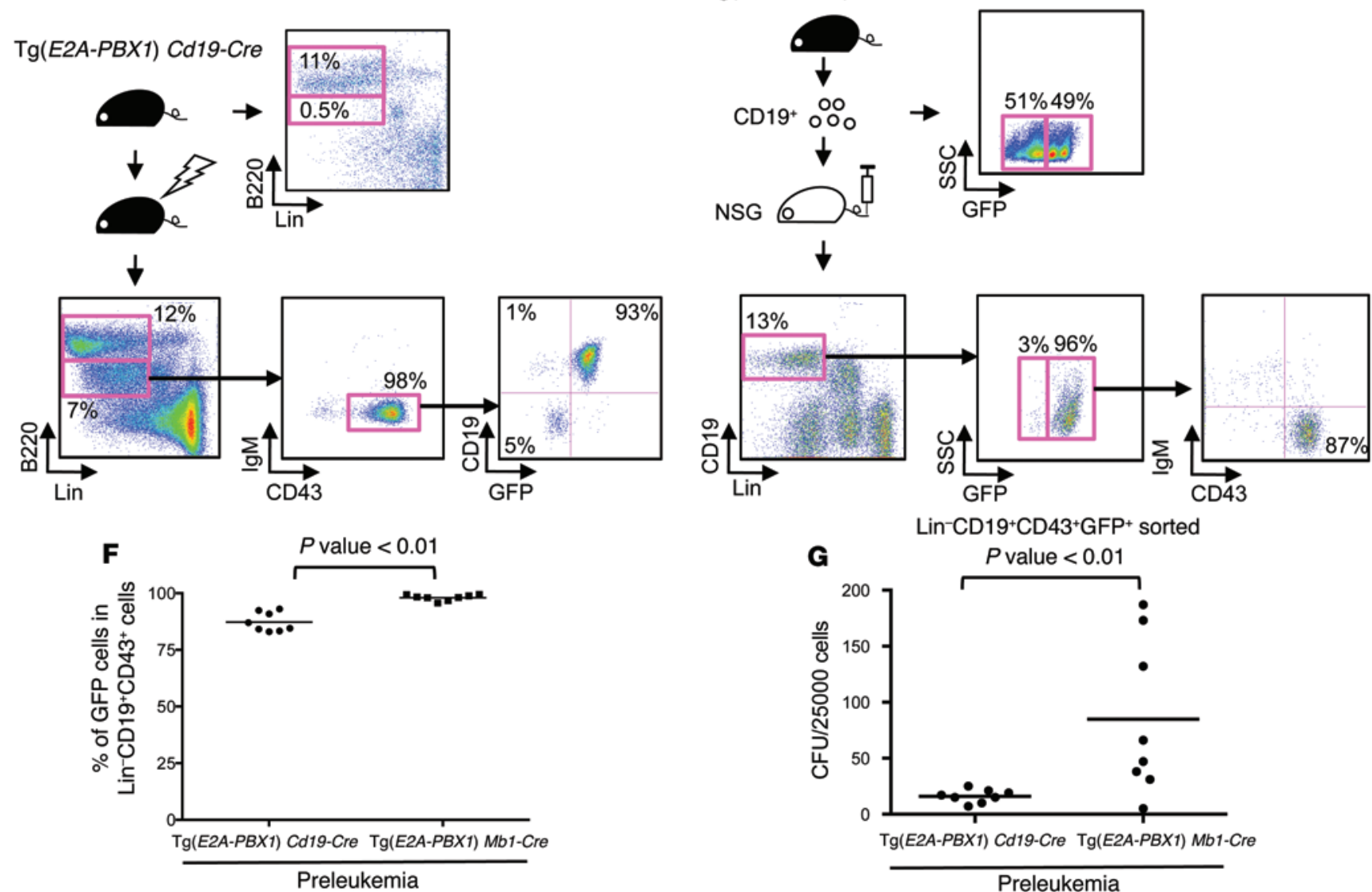

Lin-CD19+CD43+GFP+ sorted

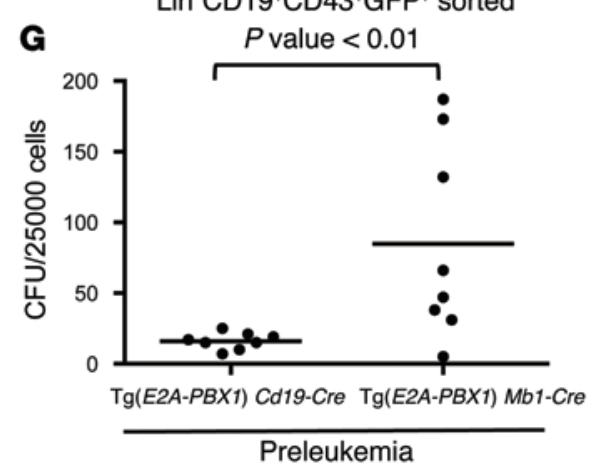

Figure 4. E2A-PBX1 confers self-renewal properties and impedes differentiation of preleukemic $B$ cell progenitors. (A) Peripheral blood B cell populations were assessed by flow cytometry at the indicated ages in control and preleukemic (\#1 and \#2) mice. (B) Bar graphs summarize B cell subset quantification following culture of prospectively isolated progenitor B cells from $\operatorname{Tg}(E 2 A-P B X 1) C d 19$-Cre preleukemic $(n=3)$ or control mice $(n=3)$. (C) Flow cytometric analysis at the end of culture for representative control and $\operatorname{Tg}(E 2 A-P B X 1)$ Cd19-Cre mice. Bar graphs summarize cell proliferation. (D) WT $(n=4)$ and preleukemic $\operatorname{Tg}(E 2 A-P B X 1) C d 19$-Cre mice $(n=4)$ were sublethally irradiated and assessed by flow cytometry for B cell progenitor recovery at 2 , 4,6 , and 8 weeks. Results at 6 weeks showed markedly skewed BM repopulation by GFP' versus control GFP- pro-B cells. Similar results were found at the different time points analyzed. (E) BM CD19+ cells (equally composed of GFP+ and GFP- cells) isolated from $\operatorname{Tg}(E 2 A-P B X 1)$ Cd19-Cre preleukemic mice were transplanted into sublethally irradiated NSG mice $(n=5)$. GFP+ B cell progenitors had a substantially enhanced engraftment and/or expansion advantage at the analyzed time points (2, 4, and 6 weeks), as shown by flow cytometry. (F) The percentage of GFP+ cells in Lin-CD19+CD43+ ${ }^{+}$cells from preleukemic $\operatorname{Tg}(E 2 A-P B X 1) C d 19-C r e(n=8)$ and $\operatorname{Tg}(E 2 A-P B X 1) M b 1-C r e(n=8)$ mice was analyzed by flow cytometry. Horizontal bars denote the mean. Statistical analysis was performed using a 2-sided Student's $t$ test. (C) Lin-CD19+CD43+CFP+ cells from the previous experiment were sorted and cultured in methylcellulose. CFU were enumerated after 7 days. Horizontal bars denote the mean. Statistical analysis was performed using a 2-sided Student's $t$ test.

Haploinsufficiency of Pax5 cooperates with E2A-PBX1 to block differentiation, shorten latency, and increase leukemia penetrance. PAX5 (BSAP) is a crucial transcription factor for B cell development (16) that is deleted in approximately $44 \%$ of pediatric ALLs with $E 2 A-P B X 1$ gene fusions $(6,7)$. Homozygous PAX5 deletions have been previously described in some pediatric ALL patients (6). Consistent with this, copy number variation (CNV) analysis of exome sequencing in 1 case (Supplemental Figure 2, A and B) and genomic quantitative PCR (qPCR) showed that $30 \%$ of leukemias harbored heterozygous or homozygous deletions of Pax 5 (Figure 
A

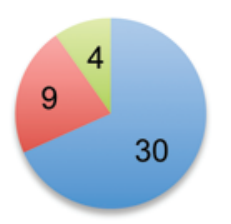

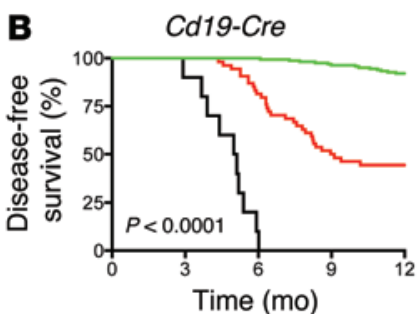
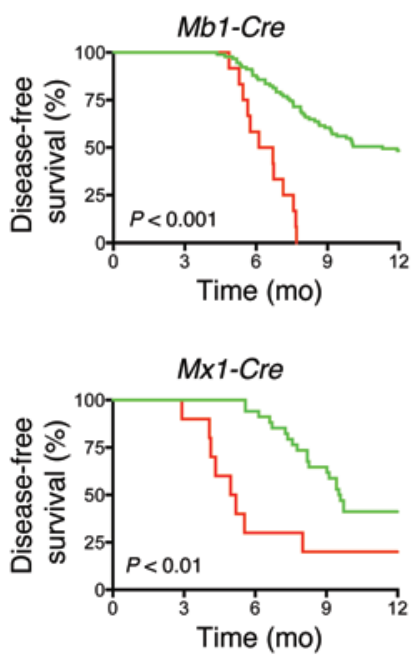

C

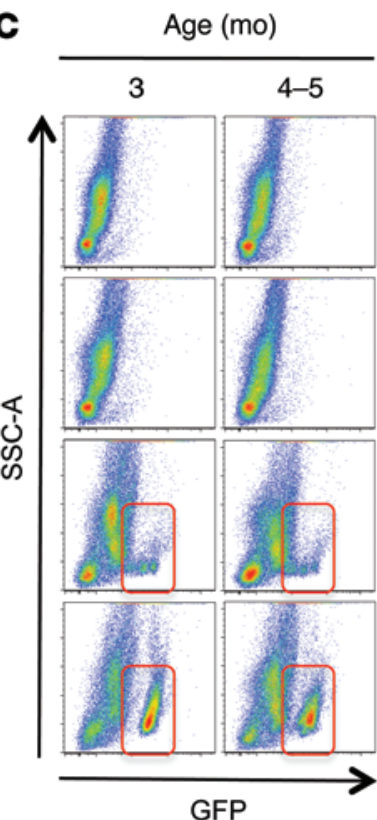

WT

Pax5 $5^{+-}$Cd19-Cre

$\operatorname{Tg}(E 2 A-P B X 1)$ Cd19-Cre

$\operatorname{Tg}(E 2 A-P B X 1) P a x 5^{+-}$Cd19-Cre

D

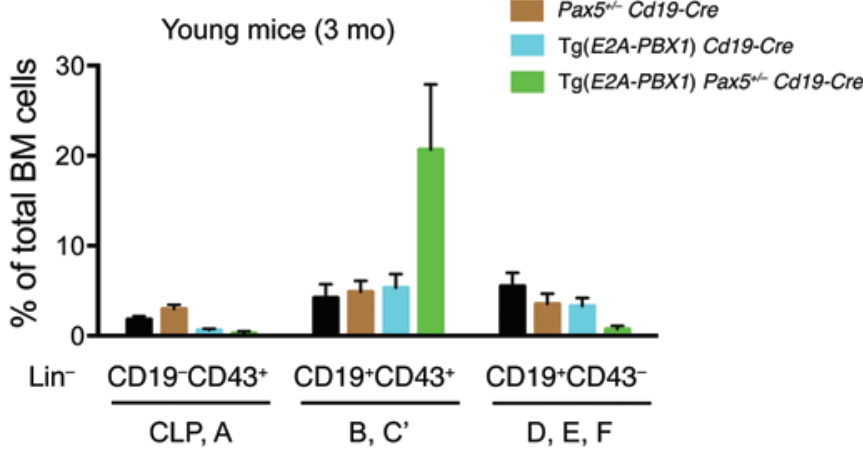

Figure 5. Haploinsufficiency of Pax5 cooperates with E2A-PBX1 to shorten latency, increase leukemia penetrance, and block differentiation. (A) Frequency of acquired Pax5 deletion in E2A-PBX1 leukemic mice $(n=43)$ as detected by genomic DNA qPCR. (B) Kaplan-Meier plots show diseasefree survival of mice. Conditional E2A-PBX1 mice were crossed with the indicated Cre-recombinase mouse line (green). Additionally, mice were crossed with conditional heterozygous Pax5-deletion mice (red; $C d 19, n=42 ; M b 1, n=12 ; M \times 1, n=9$ ) or conditional homozygous Pax5-deletion mice (black; CD19, $n=10$ ). Statistical differences were determined by log-rank test, and the survival follow-up period was 12 months. (C) Plots show flow cytometric analysis for $\mathrm{GFP}^{+}$cells in the BM of nonleukemic mice with the indicated genotypes at specific ages. Red rectangle denotes expansion of

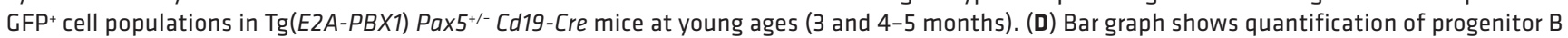
cell subpopulations in the BM of 3-month-old mice with the indicated genotypes ( $n \geq 4)$, analyzed using an Ab cocktail for lineage markers (CD3, CD4, CD8, Gr1, CD11b, NK1-1, Ter119) and CD19 and CD43 cell-surface antigens.

$5 \mathrm{~A}$ and Supplemental Figure 2, C and D) that were associated with decreased PAX5 expression (Supplemental Figure 2E).

To study the potential cooperation between E2A-PBX1 and PAX5 insufficiency, conditional E2A-PBX1 Tg mice were crossed with floxed Pax 5 mice in which deletion of exon 2 is achieved by expression of Cre recombinase (16). Heterozygous deletion of Pax 5 substantially increased the penetrance and shortened the latency of leukemia in each of the E2A-PBX1 lines, providing strong evidence for cooperative oncogenic effects of Pax5 haploinsufficiency (Figure 5B). Consistent with a tumor suppressor role, homozygous Pax5 deletion with $\operatorname{Tg}(E 2 A-P B X 1)$ Cd19-Cre background further increased the penetrance and accelerated leukemia development (Figure 5B). Pax5 deletion was confirmed in most of the BM leukemia samples analyzed by genomic qPCR (Figure 6).
Preleukemic E2A-PBX1 mice haploinsufficient for Pax 5 showed a marked expansion of $\mathrm{GFP}^{+}$progenitor $\mathrm{B}$ cells of the $\mathrm{B}-\mathrm{C}^{\prime}$ fractions (Lin $\left.{ }^{-} \mathrm{CD} 19^{+} \mathrm{CD} 43^{+}\right)$at younger ages ( 3 and $4-5$ months) compared with E2A-PBX1 Tg mice (Figure 5, C and D), which showed a slight expansion of the same progenitor $B$ cell population compared with that seen in WT mice. Hence, immature B cells of the D-E-F fractions ( $\left.\mathrm{Lin}^{-} \mathrm{CD} 19^{+} \mathrm{CD}^{-} 3^{-}\right)$were further decreased in the $\mathrm{Tg}(E 2 A-P B X 1)$ Pax5 $5^{+-}$Cd19-Cre preleukemic mice compared with WT, Pax $5^{+/-}$ Cd19-Cre, and $\operatorname{Tg}(E 2 A-P B X 1) C d 19-C r e$ control mice, indicating a more severe block at this stage of maturation that matched the leukemia phenotype. Thus, acquired insufficiency of Pax 5 is a common feature of E2A-PBX1 mice and serves to accelerate preleukemic progenitor $B$ cell expansion and development of leukemia.

Secondary mutations in murine E2A-PBX1 leukemias. To further identify cooperating mutations, single nucleotide variant (SNV) 


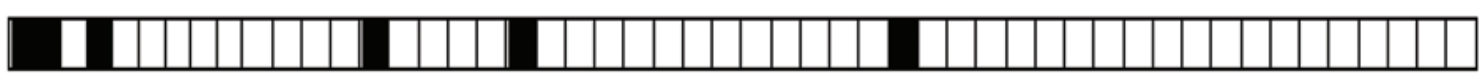

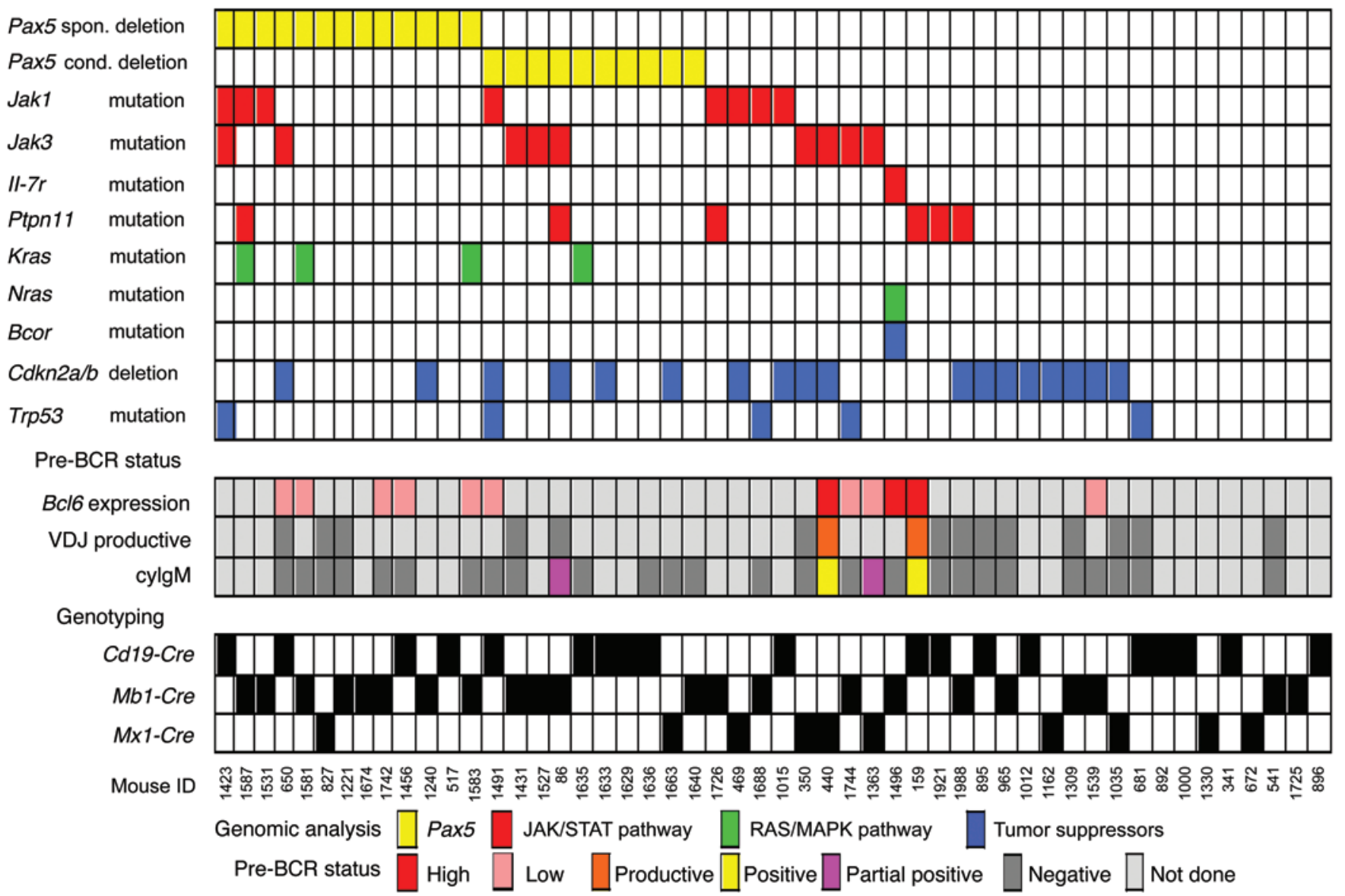

Figure 6. Secondary mutations in murine E2A-PBX1 leukemias. Genetic alterations identified in exome sequencing were validated and analyzed for recurrence in a larger cohort of leukemias $(n=51)$. Six leukemias (indicated in the top row) were analyzed by WES. All leukemias were analyzed by Sanger sequencing and genomic qPCR. Each column represents a leukemia sample and each row a genetic alteration (deletion or mutation). Identified recurrent genes were functionally grouped as follows: spontaneous and conditional Pax5 deletions (yellow), JAK/STAT signaling pathway (red), RAS/MAPK signaling pathway (green), tumor-suppressor genes (blue). Recurrent genetic alterations were found in $84.3 \%$ of leukemias. Pre-BCR status, including the presence of cytoplasmic $\mu$, productive VDJ rearrangement, and Bcl6 expression, was analyzed in selected leukemias. Mouse genotypes are depicted in the lower part of the panel. spon., spontaneous; cond., conditional.

and indel analysis of WES was performed in six E2A-PBX1 leukemias. Identified SNVs and indels after comparison with germline DNA were further filtered by excluding known SNPs or mutations of low sequence quality, unknown functional significance, or location in intronic or untranslated regions (Supplemental Figure 3, $\mathrm{A}$ and $\mathrm{B})$. The remaining mouse SNVs and indels were then compared with WES data on human hypodiploid ALL (9) or described elsewhere by targeted sequencing (17). SNVs/indels in genes that are also affected in human ALL were selected for further validation by targeted Sanger sequencing (Supplemental Table 1), and the frequency of recurrent mutations was assessed in a larger cohort $(n=51)$ of mouse E2A-PBX1 leukemias (Figure 6).

Candidate SNVs were found in a wide variety of genes but were particularly clustered in several members of the JAK/STAT and RAS/MAPK signaling pathways, suggesting that aberrant signaling pathway activation may contribute to leukemia development
(Supplemental Figure 3C). Missense Jak1 mutations localized to the pseudo-kinase domain, which has an inhibitory function on the tyrosine kinase domain (Supplemental Figure 4, A-C). Comparable mutations have been shown to confer cytokine-independent growth in vitro and are present in human ALL $(18,19)$. Mutations in the JAK/STAT pathway (Jak1, Jak3, Ptpn11, and Il7r genes) were found by targeted Sanger sequencing in 39\% of leukemias (20 of 51) (Supplemental Figure 4D). Thus, the JAK/STAT pathway was frequently mutated in $\mathrm{B}$ cell precursor leukemias induced by E2APBX1 in the conditional mouse model.

Mutations were also observed in the RAS/MAPK pathway in $20 \%$ of leukemias (10 of 51) (Supplemental Figure 4E). Comparison of survival curves, depending on RAS/MAPK mutational status, showed that mouse E2A-PBX1 leukemias with RAS/MAPK pathway mutations had significantly shorter latency compared with latencies of nonmutated leukemias ( $P=0.03$, data not shown). 
A

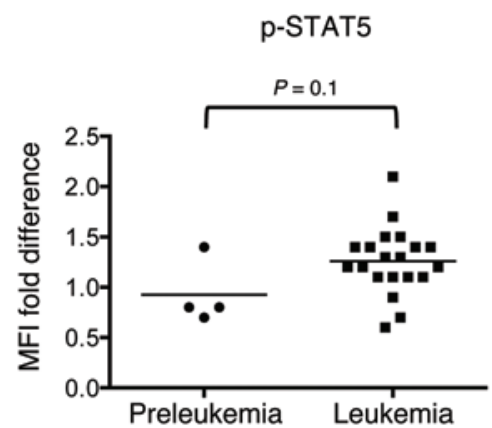

B

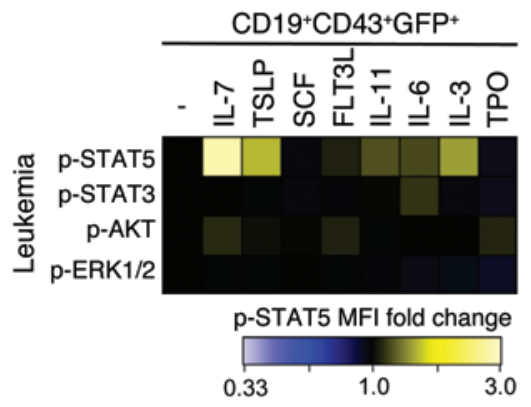

C

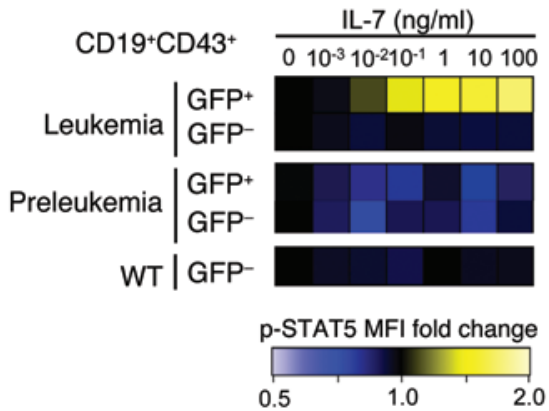

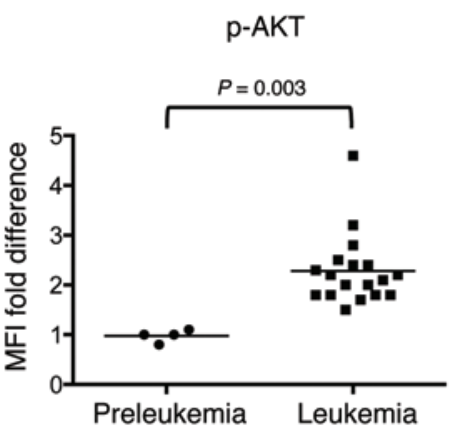

D Leukemias, $\mathrm{CD} 19^{+} \mathrm{CD}^{2} 3^{+} \mathrm{GFP}+$

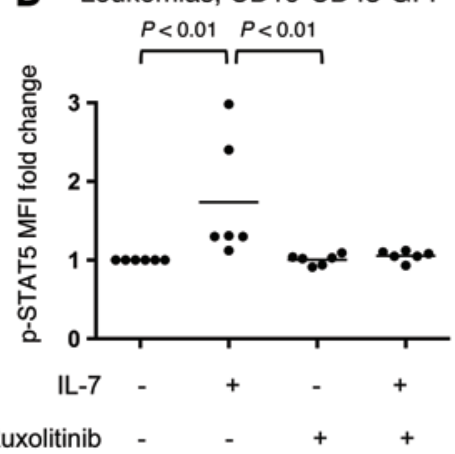

E

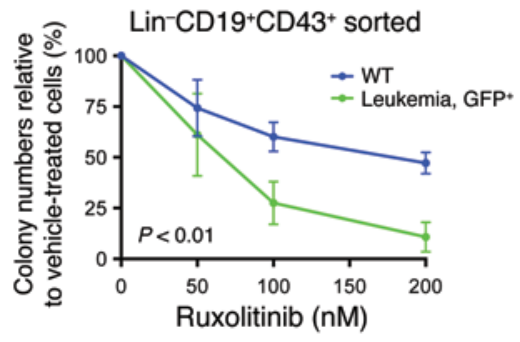

p-ERK $1 / 2$

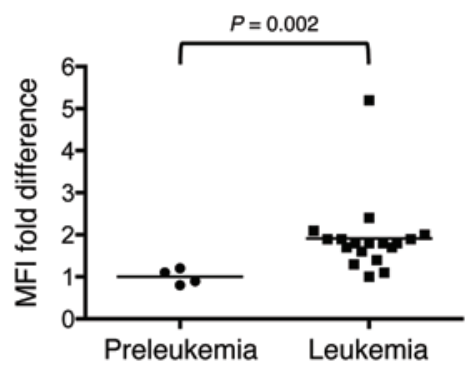

F

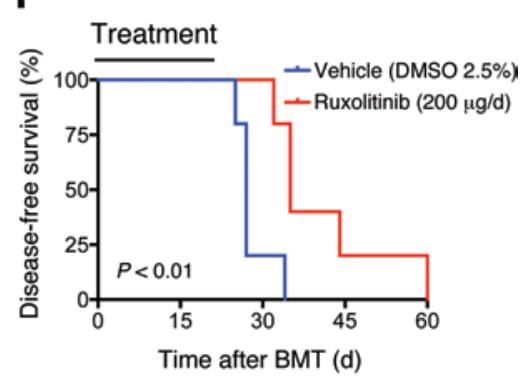

G

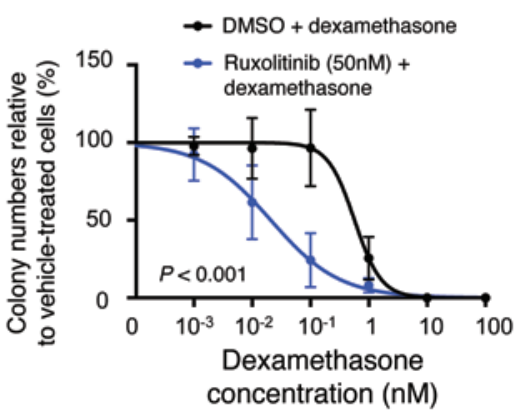

Figure 7. Aberrant activation of signaling pathways in E2A-PBX1 leukemia cells. (A) Basal phosphorylation of signaling proteins was analyzed by phospho-flow in preleukemic $(n=4)$ and leukemic E2A-PBX1 $(n=18)$ BM cells. Results are shown as the MFI of CD19+CFP+ cells compared with that of CD19+GFP- cells. Horizontal bars denote the mean. Statistical analysis was performed using a 2-sided Student's $t$ test. (B) BM leukemia cells were stimulated with cytokines, and the phosphorylation of signaling proteins was analyzed. Heatmap shows the MFI fold induction of a representative of 2 experiments. (C) BM cells were stimulated with IL-7 at increasing concentrations, and p-STAT5 was analyzed. Heatmap shows the MFI fold induction of a representative of 3 experiments. (D) BM cells were pretreated with ruxolitinib $(1 \mu \mathrm{M})$ or vehicle (DMSO) and then stimulated with IL-7 (10 ng/ $\mu \mathrm{I})$. p-STAT5 MFI was determined and compared with unstimulated/untreated cells. Results from leukemic E2A-PBX1 mice $(n=6)$ are shown. $(E)$ Lin $^{-}$CD19 ${ }^{+}$CD43 ${ }^{+}$cells from the BM of WT $(n=4)$ and leukemic E2A-PBX1 mice $(n=5)$ were FACS sorted and cultured at increasing concentrations of ruxolitinib. Colonies were enumerated, and results are expressed as the mean \pm SEM. Statistical analysis was performed using the F test. (F) E2A-PBX1 leukemia cells with a JAK1 L652E mutation were transplanted into sublethally irradiated secondary recipient mice ( $n=5$ in each cohort). Mice were treated with either vehicle or ruxolitinib $(200 \mu$ g, i.p.) daily for 20 days. Statistical analysis was performed using the log-rank test. (G) Dose-response curves are shown for leukemia cells $(n=4)$ cultured in the presence of vehicle or ruxolitinib at increasing concentrations of dexamethasone. Colonies were enumerated, and results are expressed as the mean \pm SEM. Statistical analysis was performed using the $\mathrm{F}$ test.

$C D K N 2 a / b$ locus deletions were reported in 9 of 22 of E2APBX1 leukemia patients (20). CNV analysis of mouse E2A-PBX1 leukemia WES showed deletions of the $C d k n 2 a / b$ locus in 2 of 6 leukemias (Supplemental Figure 5A). Genomic qPCR of a larger cohort detected $15 \%$ and $25 \%$ of leukemias with heterozygous and homozygous $C d k n 2 a / b$ deletions, respectively (Supplemental Figure 5, B and C). Decreased expression of Cdkn2a and Cdkn2b transcripts correlated with deletion of the $C d k n 2 a / b$ locus in sorted
$\mathrm{Lin}^{-} \mathrm{CD} 19^{+} \mathrm{CD} 3^{+} \mathrm{GFP}^{+}$leukemia cells (Supplemental Figure 5D).

Trp53 mutations affecting its DNA-binding domain were detected by WES in 2 of 6 mouse leukemias (Supplemental Figure $\left.6, \mathrm{~A}^{-} \mathrm{C}\right)$ and by targeted Sanger sequencing in 5 of $51(10 \%)$ in a larger cohort (Supplemental Figure 6D), consistent with previously described TP53 mutations in E2A-PBX1+ patients $(21,22)$.

Inhibition of signaling pathways activated by secondary point mutations impairs E2A-PBX1 leukemia cell growth in vitro and in vivo. 
The observed acquired mutations suggested possible activation of specific signaling pathways in E2A-PBX1 leukemia cells. Phosphoflow analysis revealed higher basal levels of phosphorylated STAT5 (p-STAT5), p-AKT, and p-ERK1/2 in leukemia versus preleukemic cells (Figure 7A), consistent with acquired activation of the respective signaling pathways. Compared with preleukemic and WT $\mathrm{CD} 19^{+} \mathrm{CD} 43^{+} \mathrm{B}$ cell progenitors, leukemia cells also displayed hypersensitivity to stimulation with IL-7, as measured by induction of p-STAT5 (Figure 7, B and C). IL-7 supported growth in colonyforming assays (Supplemental Figure 7A) of leukemia cells, which displayed a more robust clonogenic response than did WT and preleukemic cells. Thus, mouse E2A-PBX1 leukemia cells display functional and genetic evidence for IL-7R/JAK/STAT signaling pathway activation.

To characterize the role of the JAK/STAT pathway in cell proliferation, we used the specific JAK inhibitor ruxolitinib, which is approved for treatment of myeloproliferative disorders $(23,24)$ and blocks the activation of WT as well as mutant JAKs $(25,26)$. Ruxolitinib blocked the induction of p-STAT5 by IL-7 stimulation of mouse E2A-PBX1 leukemias (Figure 7D) as well as of human primary ALL and ALL cell lines (Supplemental Figure 7, B and C). A potential therapeutic window was assessed by cultures of sorted normal WT progenitor B cells and leukemic E2A-PBX1 cells in the presence of different concentrations of ruxolitinib. Colony formation in vitro of both WT and leukemic B cell progenitors was inhibited, however leukemic cells were more sensitive, with an $\mathrm{IC}_{50}$ of $62 \mathrm{nM}$, whereas WT progenitor B cells did not reach an $\mathrm{IC}_{50}$ at 200 $\mathrm{nM}$ (Figure 7E). A role for the JAK/STAT pathway inhibition in leukemia cell proliferation was assessed in vivo. Mice transplanted with E2A-PBX1 leukemia cells with a JAK1 L652E mutation treated with ruxolitinib showed a statistically significant increase in disease-free survival (median 35 days vs. 27 days, $P=0.007$ ), with no observed toxicities (Figure $7 \mathrm{~F}$ ).

Ruxolitinib sensitized mouse E2A-PBX1 leukemia cells to the growth inhibitory effect of chemotherapy. Leukemia cells cultured in the presence of ruxolitinib at increasing doses of dexamethasone had a lower $\mathrm{IC}_{50}$ compared with that of vehicle-treated cells (Figure $7 \mathrm{G}, P=0.001$ ), suggesting a rationale for combining JAK inhibitors with chemotherapy in the treatment of ALL. These results indicate that an activated JAK/STAT pathway is crucial for cell proliferation and survival in vitro and in vivo and might be targeted by small-molecule inhibitors in E2A-PBX1 leukemias.

\section{Discussion}

We investigated the translational biology of B cell precursor ALL using comparative genomics and functional approaches. Our results experimentally recapitulate the multistep pathogenesis of ALL previously inferred from genomic analyses and highlight key cooperating oncogenic pathways. Using a novel E2A-PBX1 mouse model as a preclinical platform, the therapeutic efficacy was established for targeting the pre-BCR and JAK/STAT pathways in a distinctive genetic subtype of ALL.

Conditional activation of E2A-PBX1 in the B cell compartment of mice consistently induces ALLs that are similar to human $\mathrm{E} 2 \mathrm{~A}-\mathrm{PBX} 1^{+}$leukemias, in contrast to alternative models for $\mathrm{E} 2 \mathrm{~A}$ $P B X 1$ oncogene expression (27-30). The leukemias are composed of lymphoblasts with phenotypic profiles and maturational arrest that are very similar to those of the pro-B/large pre-B II stage seen in human E2A-PBX1 leukemia patients. A distinctive feature of human E2A-PBX1 leukemias is the presence of cytoplasmic $\mu$ chain in up to $80 \%$ of cases $(31,32)$ and functional pre-BCR (13). Cytoplasmic $\mu$ chain with concomitant surface VPREB expression were also seen in a subset of mouse leukemias, albeit at a lower percentage (12.5\%), likely due to a higher frequency of nonproductive VDJ rearrangements. These observations indicate that pre-BCR signaling is not absolutely required for $\mathrm{B}$ cell progenitor transformation by E2A-PBX1. However, pre-BCR ${ }^{+}$E2A-PBX1 leukemias displayed notable functional differences including high Bcl6 expression, response to pre-BCR stimulation, and sensitivity to dasatinib in vitro and in vivo, very similar to recently reported data on human pre-BCR ${ }^{+}$ALL that included patients with E2A$\mathrm{PBX}^{+}$leukemia (13). Consistent with this, conversion of pre-BCR ${ }^{-}$ to pre- $\mathrm{BCR}^{+}$leukemias by overexpression of a functional $\mu$ heavy chain conferred dasatinib sensitivity. Leukemias that differed according to pre-BCR status were otherwise similar immunophenotypically, and, in fact, mixtures of cyIgM ${ }^{-}$and $\operatorname{cyIgM}^{+}$leukemic blasts were detected in some leukemias. Thus, the E2A-PBX1 mouse model induces functionally distinct pre- $\mathrm{BCR}^{+}$and pre$\mathrm{BCR}^{-}$leukemias with maturational arrest at the pro-B/large pre- $\mathrm{B}$ II stages of B cell differentiation, recapitulating human E2A-PBX1 leukemia pathobiology.

One pre-BCR- leukemia displayed discordantly high levels of Bcl6 associated with a Bcor disruptive mutation (K1179*), analogous to BCOR mutations described in human ALL (9) and acute myeloid leukemia (AML) (33). Notably, Bcor-mutant leukemia cells were relatively insensitive to dasatinib in vitro. The implication of Bcl6 upregulation independent of pre-BCR signaling as a clinical mechanism for dasatinib resistance requires further investigation.

The conditional expression of E2A-PBX1 in B cell progenitors not only contributes to a block of differentiation but also provides proliferative advantage, suggesting that E2A-PBX1 confers self-renewal properties to lymphoid progenitors, as already postulated from human ALL. Enhanced self-renewal would promote the extended longevity of B cell precursors to acquire and accumulate additional genomic aberrations and secondary mutations, which collaborate to fully transform these B cell precursors into leukemia cells.

In support of this scenario, mouse E2A-PBX1 leukemias harbor various somatically acquired genetic aberrations. Most notably, this includes Pax 5 deletions, which appear to be part of the natural history of the mouse leukemias (Figure 5A) and have been observed in $44 \%$ of human E2A-PBX1 leukemias. However, their pathologic contributions in cooperation with E2A-PBX1 have not been previously demonstrated. Intercrossing of conditional E2A-PBX1 mice with conditional Pax5-deletion mice resulted in markedly expanded progenitor B cell subpopulations in healthy preleukemic mice and substantial acceleration of leukemia development with increased penetrance. The observed gene dosage effects establish a tumor-suppressor role for Pax 5 on an E2A-PBX1 background (Figure 5B). Our results provide, to our knowledge, the first experimental evidence for a strong cooperative role of Pax5 insufficiency in E2A-PBX1 leukemogenesis and confirm the role of Pax 5 loss in enhancing the maturational arrest and proliferative expansion of B cell progenitors initiated by E2A-PBX1. 
Similar to our findings, Pax 5 haploinsufficiency has recently been described to inhibit B cell differentiation and to increase leukemia penetrance in a Tg B-ALL mouse model driven by a constitutively active form of STAT5 (34).

No other genetic aberrations in essential transcription factors such as Ebf1, Ikfz1, or Ikfz3, which are required for B cell development, were detected in $\mathrm{CNV}$ and SNV analyses of exome sequences in mouse leukemias. However, E2A-PBX1 leukemia cells are, by default, haploinsufficient for $E 2 A$ as a consequence of the $\mathrm{t}(1 ; 19)$ translocation, which may contribute to E2A-PBX1 leukemia pathogenesis, given that E2A deletions are present in a small subset of non-E2A-PBX1 human leukemias (6, 35 ). Although the potential role of E2A haploinsufficiency was not directly assessed in this study, we hypothesize that it is a key feature of our conditional E2A-PBX1 mouse model, wherein simultaneous activation of E2A-PBX1 and haploinsufficiency of E2A may contribute to leukemia incidence and representativeness of human E2A-PBX1 ALL.

Our studies highlight a prominent loss of other tumor suppressors in E2A-PBX1 ALL. CNV analysis of a large cohort of mouse E2A-PBX1 leukemias showed deletions of the $C d k n 2 a / b$ locus in $40 \%$ of these leukemias, which matches the frequency (41\%) observed in human patients (20). Furthermore, Trp53 mutations were observed in 5 of 51 conditional mouse leukemias, which is similar to the frequency observed in E2A-PBX1 ${ }^{+}$leukemias (21, 22). Mutations and deletions in the TP53 gene in human ALLs are uncommon $(2 \%-3 \%)$ at first diagnosis, but present at higher frequencies (12\%-36\%) in relapsed BM samples $(36,37)$.

WES identified specific signaling pathways vulnerable to acquired mutations in E2A-PBX1 leukemias. Most prominently, mutations in key components of the JAK/STAT pathway, including Jak1 (exon 14) and Jak3 (exon 15) genes, were found in 39\% of mouse E2A-PBX1+ leukemias. Although JAK2 R683 is the most frequent JAK mutation in human ALL, it was not observed by Sanger sequencing of Jak2 (exon 15) in mouse leukemias (data not shown). Since there was no correlation between JAK/STAT pathway mutational status and p-STAT5 basal levels, IL-7/p-STAT5 stimulation, or disease-free survival, we hypothesize that additional mechanisms might regulate the JAK/STAT5 pathway in mouse E2APBX1 leukemia cells not detected in our genomic analyses.

Although JAK1/2/3 mutations are present in human ALL subtypes, noJAK1/2/3 mutationshaveyetbeenobserved inhumanE2APBX1 leukemias using targeted sequencing technologies $(19,38)$. Nevertheless, we observed IL-7/p-STAT5 induction in leukemic $\mathrm{BM}$ cells from two $\mathrm{E} 2 \mathrm{~A}-\mathrm{PBX} 1^{+}$patients at similar or higher levels compared with non-E2A-PBX1 patients. Increased expression of $I L 7 R$ and decreased expression of the negative pathway regulators $S H 2 B 3 / L N K$ and SOCS2 were detected in $\mathrm{E} 2 \mathrm{~A}-\mathrm{PBX} 1^{+}$ compared with E2A-PBX1- patients from 2 large, independent cohorts $(39,40)$ with publicly available data (Supplemental Figure 8). Although, genomic analyses of mouse E2A-PBX1 leukemias strongly support a cooperative role for the JAK/STAT pathway, clarification of other possible mechanisms for pathway activation in human E2A-PBX1+ leukemias is needed. WES studies in human E2A-PBX1 ${ }^{+}$ALL or targeted sequencing of PTPN11 and IL7R genes might reveal JAK/STAT pathway mutations in E2A-PBX1 $1^{+}$leukemias. A more detailed characterization of JAK/STAT pathway acti- vation and demonstration of the preclinical efficacy of ruxolitinib in human E2A-PBX1 leukemias are necessary to further assess the importance of this pathway.

Conversely, activating mutations in Nras and Kras may lead to activation of the RAS/MAP kinase pathway, as seen by increased p-ERK basal levels in leukemia cells. Increased p-AKT levels may highlight the cross-talk between the RAS/MAP kinase and PI3K/ AKT pathways (41). Of note, although NRAS and KRAS mutations have not been described at diagnosis in E2A-PBX1 patients by targeted sequencing, the three $\mathrm{E} 2 \mathrm{~A}-\mathrm{PBX} 1^{+}$human cell lines used in this study and analyzed in the Cancer Cell Line Encyclopedia (42) harbor mutations in this pathway.

The extensive similarities between the conditional E2A-PBX1 Tg mouse model and human E2A-PBX1+ ALL provide a platform for preclinical studies of drug screening and development. Targeting pre-BCR signaling by dasatinib and the JAK/STAT pathway by ruxolitinib in pre-BCR ${ }^{+}$and JAK1-mutated leukemias, respectively, extended disease-free survival in secondary transplantation experiments but did not completely inhibit the growth of leukemia cells. The mechanisms of resistance to pre-BCR signaling and JAK/STAT pathway inhibition in E2A-PBX1 ALL require further investigation. Nevertheless, these results suggest that dasatinib and ruxolitinib might have clinical efficacy, particularly in this ALL subtype and generally in human ALL.

In summary, comparative genomic and functional analyses identified 2 distinct subtypes of E2A-PBX1+ ALL that differ on the basis of their pre-BCR status. Mouse E2A-PBX1+ leukemias show consistent loss of tumor-suppressor genes (Pax5 and $C d k n 2 a / b)$ and activation of signaling pathways by point mutations (JAK/ STAT, RAS/MAPK). Conditional Tg activation of E2A-PBX1 confirmed the multistep etiology of ALL previously inferred from genomic analyses and provided a platform for preclinical studies establishing the efficacy of targeting pre-BCR signaling by dasatinib and the JAK/STAT pathway by ruxolitinib.

\section{Methods}

Mice. A conditional E2A-PBX1 allele was engineered to recombine human $P B X 1 a$ cDNA into the mouse E2A locus to create an E2A-PBX1 fusion gene. Using a previously reported $E 2 A$ conditional $\mathrm{KO}$ construct (43) as a template, a PGK neocassette was positioned immediately downstream of the E2A gene, followed by a loxP site, a splice acceptor sequence, and human PBX1a 3' cDNA sequences linked by an IRES element with the EGFP coding region. The targeted $E 2 A$ allele encodes WT $E 2 A$, but Cre-mediated recombination repositions the downstream $P B X 1$-EGFP cassette into the $E 2 A$ gene, inducing expression of an $E 2 A$ $P B X 1$ fusion transcript and EGFP under control of the E2A promoter. Tg Cd19-Cre (The Jackson Laboratory, ref. 44), Mx1-Cre (The Jackson Laboratory, ref. 45), and Mb1-Cre (provided by David Allman, University of Pennsylvania, Philadelphia, Pennsylvania, USA, ref. 46) mice were intercrossed to generate $\operatorname{Tg}(E 2 A-P B X 1) C d 19-C r e, \operatorname{Tg}(E 2 A-P B X 1)$ $M x 1-C r e$, and $\operatorname{Tg}(E 2 A-P B X 1) M b 1-C r e$ mice, respectively, on a C57BL/6 background. Floxed Pax5-deletion mice (provided by Meinrad Busslinger, Research Institute of Molecular Pathology, Vienna, Austria) (16) were crossed with conditional E2A-PBX1 and Cre recombinase mouse lines. The Mx1-Cre promoter was leaky, and polyIC was not necessary to induce Cre recombinase. $\mathrm{GFP}^{+}$cells were present in the HSC compartment $\left(\mathrm{Lin}^{-} \mathrm{Sca1}^{+} \mathrm{Kit}^{+}\right)$of $\operatorname{Tg}(E 2 A-P B X 1)$ Mx1-Cre mice. Mutant and 
control mice were genotyped by PCR. NSG mice were obtained from The Jackson Laboratory.

Histology and cytology. Tissues were fixed in $10 \%$ formalin, embedded in paraffin, and sectioned and stained with H\&E. Blood smears were fixed with $100 \%$ methanol and stained with May-Grünwald solution (Sigma-Aldrich) and Giemsa (Sigma-Aldrich) solution according to the manufacturers' instructions.

Human cell lines. Human leukemia cell lines 697, RCH-ACV, Kasumi-2, RS4-11, and REH (obtained from Leibniz-Institut DSMZ) were cultured in RPMI 1640 medium supplemented with $10 \%$ FBS, 100 $\mathrm{U} / \mathrm{ml}$ penicillin-streptomycin, and $0.29 \mathrm{mg} / \mathrm{ml} \mathrm{L}$-glutamine. SUP-B15 cells (obtained from ATCC) were cultured in RPMI 1640 medium supplemented with $20 \%$ FBS. E2A-PBX1 ${ }^{+}$cell lines were authenticated using Western blot analysis for fusion protein expression.

Flow cytometry and FACS. Cells were flushed from large bones or dissected from spleen and lymph nodes, filtered, rid of rbc in $1 \times$ rbc lysis buffer (eBioscience), then washed twice with PBS. Brain and spinal cord were minced, treated with Accutase (STEMCELL Technologies) at $37^{\circ} \mathrm{C}$ for 30 minutes, and homogenized by passage through a 16-gauge needle. Cells were washed twice with staining buffer (PBS and $0.5 \% \mathrm{FBS}$ ), resuspended, incubated with Abs, and washed again with staining buffer before acquisition of data in a flow cytometer. For intracellular staining, cells were fixed with $1.5 \%$ formaldehyde for 10 minutes at room temperature, permeabilized with $100 \%$ icecold methanol for 20 minutes on ice, and washed twice with staining buffer. Flow cytometry was performed in an LRS Fortessa (BD Biosciences) using FACSDiva (BD Biosciences) and FlowJo software for analysis. Cells were sorted for cell-surface markers using a FACSAria cell sorter (BD Biosciences). Abs used for flow cytometric analysis and FACS sorting are listed in Supplemental Table 2.

Phospho-flow analysis. Murine cells were serum starved for 1 hour at $37^{\circ} \mathrm{C}$ in DMEM high-glucose medium (Thermo Scientific) containing $0.1 \% \mathrm{FBS}$, then stimulated for 30 minutes at $37^{\circ} \mathrm{C}$ using the following reagents to stimulate pre-BCR signaling (15): $625 \mu \mathrm{M}$ sodium pervanadate (Sigma-Aldrich); $5 \mu \mathrm{g} / \mathrm{ml}$ goat (SouthernBiotech) and 5 $\mu \mathrm{g} / \mathrm{ml}$ rat polyclonal anti-mouse IgM (BD Biosciences); and $33 \mu \mathrm{M}$ $\mathrm{H}_{2} \mathrm{O}_{2}$ (Sigma-Aldrich). The following murine cytokines were used for 30 minutes at $37^{\circ} \mathrm{C}$ to stimulate mouse leukemia cells: $10 \mathrm{ng} / \mathrm{ml}$ IL-7 (PeproTech); $10 \mathrm{ng} / \mathrm{ml}$ TSLP (R\&D Systems); $100 \mathrm{ng} / \mathrm{ml}$ SCF (PeproTech); $100 \mathrm{ng} / \mathrm{ml}$ FLT3L (PeproTech); $100 \mathrm{ng} / \mathrm{ml}$ IL-11 (PeproTech); 100 ng/ml IL-6 (PeproTech); 100 ng/ml IL-3 (PeproTech); or 500 ng/ml TPO (PeproTech).

Human cell lines maintained in exponential growth were serum starved overnight at $37^{\circ} \mathrm{C}$ in RPMI 1640 medium containing $0.1 \%$ FBS, then stimulated for 30 minutes with $10 \mathrm{ng} / \mathrm{ml}$ human IL-7 (BD Pharmingen). Primary human ALL samples were obtained from the Tissue Bank of the Stanford University Department of Pediatrics. Cells were thawed, incubated for 1 hour at $37^{\circ} \mathrm{C}$, then stimulated with $10 \mathrm{ng} / \mathrm{ml}$ human IL-7 for 30 minutes.

Murine as well as primary human cells and human cell lines were pretreated with ruxolitinib (INCB018424; Selleckchem) or vehicle (DMSO) for 15 minutes prior to cytokine stimulation.

Cells were subsequently fixed and permeabilized as described previously $(47,48)$ and stained with conjugated Abs against intracellular phospho-proteins (see Supplemental Table 3) and cellsurface markers. Data were analyzed using the Cytobank web-based platform (49).
BM transplantation assays. Secondary transplantation of BM cells $\left(5 \times 10^{5}\right)$ from leukemic mice was performed by retro-orbital injection after sublethal irradiation (4.5 Gy) of 8- to 12-week-old C57BL/6 mice. Moribund mice were sacrificed, and leukemia was confirmed by May-Grünwald Giemsa staining of blood smears and the presence of $\mathrm{GFP}^{+}$cells by FACS analysis.

For the in vivo-treatment experiments, 1,000 BM cells from leukemic mice were transplanted retro-orbitally. Mice were treated daily, i.p., for 20 days, starting the day after transplantation with $100 \mu \mathrm{g}$ dasatinib (LC Laboratories), $100 \mu \mathrm{g}$ ruxolitinib, or vehicle (for dasatinib, 30\% PEG1500, 1\% Tween 80, 2.5\% DMSO dissolved in PBS; for ruxolitinib, 2.5\% DMSO dissolved in PBS).

Colony-forming assays. Leukemia cells were cultured in methylcellulose medium (IL-7-containing MethoCult M3630 or M3234; STEMCELL Technologies) supplemented with $10 \mathrm{ng} / \mathrm{ml}$ IL-7 (Miltenyi Biotec), 100 ng/ml SCF (PeproTech), and 100 ng/ml FLT3L (PeproTech) unless otherwise indicated (Figure 7 and Supplemental Figure 7). Leukemia cells were treated with dasatinib, ruxolitinib, dexamethasone (Sigma-Aldrich), or vehicle (DMSO) at the indicated concentrations (Figures 3 and 7).

PCR. Genomic DNA was amplified in a PTC-100 Peltier thermal cycler (MJ Research), and PCR products were separated by gel electrophoresis and visualized by ethidium bromide staining. For real-time qPCR, isolated DNA was amplified with specific primers (Supplemental Tables 4 and 5 for Pax 5 and $C d k n 2 a / b$ deletions, respectively) using an ABI 7900HT thermal cycler with SYBR Green (Applied Biosystems) with an annealing temperature of $60^{\circ} \mathrm{C}$. All signals were quantified using the $\Delta \mathrm{Ct}$ method and normalized to $\Delta \mathrm{Ct}$ values of the transferrin receptor (Tfrc) gene. Samples from different qPCR assays were subsequently normalized to WT BM samples.

qPCR. RNA was isolated using the RNeasy Mini Kit (QIAGEN), and cDNA was synthesized using the SuperScript III Reverse Transcriptase Kit (Life Technologies) according to the manufacturer's recommendations. Relative Cdkn2a and Cdkn2b expression was quantified using the ABI 7900HT Thermal Cycler with TaqMan Master Mix (Applied Biosystems) at an annealing temperature of $60^{\circ} \mathrm{C}$ and the following TaqMan gene expression assays from Life Technologies: Cdkn2a (Mm00494449_m1), Cdkn2b (Mm00483241_m1), and Actb (Mm00607939_s1). Relative Bcl6 expression was quantified using SYBR Green Master Mix and primers for Bcl6 (CCGGCACGCTAGTGATGTT and TGTCTTATGGGCTCTAAACTGCT) and Actb (CGTGAAAAGATGACCCAGATC and CACAGCCTGGATGGCTACGT). All signals were quantified using the $\Delta \mathrm{Ct}$ method and normalized to the $\Delta \mathrm{Ct}$ values of $A c t b$ gene expression levels.

DNA sequencing and analysis. WES was performed commercially (Centrillion Biosciences), with an average yield of $11.6 \mathrm{~Gb}$ per sample. Raw sequences were aligned to the mouse genome build mm10 using BWA software.

For CNV analysis, we used Nexus CNV 7.5 software (BioDiscovery). The SNP-FASST2 segmentation algorithm and default settings for significance of CNV, number of probes per segment, and gain and loss thresholds were used (maximum contiguous probe spacing [Kbp] 1,000 , minimum number of probes per segment 3 , high gain 0.6 , gain 0.2 , loss -0.2 , big loss -1.0 ).

SNP and indel analysis was performed using PICARD (http:// picard.sourceforge.net), SAMtools (50), and GATK (51). SNPs and indels were further analyzed for functional annotations using SNPeff 
(52) and Ensembl Variant Effect Predictor (VEP) (53). Data visualization was performed using integrative genomic viewer software (IGV) (54). Functional annotation clustering of identified SNVs in WES was performed using the DAVID (Database for Annotation, Visualization and Integrated Discovery) bioinformatics resource tools $(55,56)$

For Sanger sequencing, target DNA was amplified with specific primers (Supplemental Table 6 for mutations and Supplemental Table 7 for VDJ rearrangement) and Accuprime Pfx DNA polymerase (Life Technologies) using a 2-step PCR reaction. PCR products were purified and sequenced commercially (Sequetech). Sequences from SNPs and indels were analyzed using Sequencher version 5.2 sequence analysis software (Gene Codes Corporation). Sequences from VDJ rearrangement products were analyzed using the IMGT/V-QUEST tool (57).

Western blot analysis. Proteins were isolated using a modified radioimmunoprecipitation assay (RIPA) lysis buffer containing 50 $\mathrm{mM}$ Tris $\mathrm{HCl}, 1 \% \mathrm{NP}-40,1 \%$ natrium-deoxycholate, $150 \mathrm{mM} \mathrm{NaCl}, 1$ mM EDTA, $1 \mathrm{mM}$ PMSF, $1 \mathrm{mM} \mathrm{Na} \mathrm{VO}_{4}$, and $1 \mathrm{x}$ Protease inhibitor cocktail (Roche). Equal amounts of protein were electrophoresed through $4 \%$ to $12 \%$ Bis-Tris gels, transferred to Hybond-P membranes (Amersham), and immunodetected with rat anti-E2A (clone 826927; R\&D Systems), rat anti-IgM (clone II/41; BD Biosciences), or rabbit antiGAPDH (catalog G9545; Sigma-Aldrich) Abs. Bands were detected by chemiluminescence using the ECL Plus Western Blotting Detection System and HyperFilm (Amersham). Quantification by densitometry was performed using Image J software (NIH) (58).

Cloning and $\mu$ heavy chain overexpression. cDNA of $\mu$ heavy chain from mouse leukemia with productive VDJ rearrangement was amplified using the following primers: ATGAAATGCAGCTGGGTCATCTTCTTCCTG and TCACCTCCCCCTATAGCAGGTGCCGCCTGTGTCAG. PCR products were isolated and cloned into the MSCV-Puro vector using the In-Fusion HD Cloning Kit (Clontech). Retroviruses were generated in Phoenix-Eco cells. The resulting virus supernatants were supplemented with $8 \mu \mathrm{g} / \mathrm{ml}$ polybrene (catalog sc-134220; Santa Cruz Biotechnology Inc.) for transduction of mouse leukemia cells by spinoculation $\left(1,200 \mathrm{~g}, 32^{\circ} \mathrm{C}\right.$ for 3 hours). After puromycin selection $(1 \mu \mathrm{g} / \mathrm{ml})$, transduced leukemia cells were used for experiments.

Statistics. Survival curves were analyzed by long-rank test. Statistical differences between 2 groups were analyzed with a 2-sided Student's $t$ test, assuming a normal distribution. Dose-response curves were compared using the extra sum-of-squares F test. A $P$ value of less than 0.05 was considered statistically significant. Statistical analysis was performed using GraphPad Prism software, version 6 (GraphPad Software).

Study approval. All experiments using mice were performed with the approval of and in accordance with the guidelines of the Administrative Panel on Laboratory Animal Care of Stanford University.

\section{Acknowledgments}

We thank Maria Ambrus for technical assistance, Wendy Fantl and Kara Davis for helpful discussions on phospho-flow, Ryan Corces-Zimmerman for assistance with exome-sequencing analysis, and Steve Hunger and members of the Cleary laboratory for helpful discussions. J. Duque-Afonso and F. Scherer were supported by grants DU 1287/2-1 and SCHE 1870/1-1, respectively, from the German Research Foundation (Deutsche Forschungsgemeinschaft). S.H.K. Wong was supported by a Young Investigator Award from the Alex's Lemonade Stand Foundation for Childhood Cancer. M.L. Cleary and J. Feng were supported by the Lucile Packard Foundation for Children's Health, the Child Health Research Institute, and the Stanford NIH-NCATS-CTSA grant UL1 TR001085.

Address correspondence to: Michael L. Cleary, Lokey Stem Cell Research Building, 265 Campus Drive, Stanford, California 943055457, USA. Phone: 650.723.7975; E-mail: mcleary@stanford.edu.
1. Nowell P, Hungerford D. A minute chromosome in chronic granulocytic leukemia. Science. 1960;142:1497.

2. Rowley JD. A new consistent chromosomal abnormality in chronic myelogenous leukaemia identified by quinacrine fluorescence and Giemsa staining. Nature. 1973;243(5405):290-293.

3. Rowley JD. Editorial: The role of cytogenetics in hematology. Blood.1976;48(1):1-7.

4. Kelly LM, Gilliland DG. Genetics of myeloid leukemias. Annu Rev Genomics Hum Genet. 2002;3:179-198.

5. Mullighan CG. The molecular genetic makeup of acute lymphoblastic leukemia. Hematology Am Soc Hematol Educ Program. 2012;2012:389-396.

6. Mullighan CG, et al. Genome-wide analysis of genetic alterations in acute lymphoblastic leukaemia. Nature. 2007;446(7137):758-764.

7. Mullighan CG, et al. Deletion of IKZF1 and prognosis in acute lymphoblastic leukemia. $N$ Engl J Med. 2009;360(5):470-480.

8. Roberts KG, et al. Genetic alterations activating kinase and cytokine receptor signaling in highrisk acute lymphoblastic leukemia. Cancer Cell. 2012;22(2):153-166.

9. Holmfeldt L, et al. The genomic landscape of hypodiploid acute lymphoblastic leukemia. Nat Genet. 2013;45(3):242-252.
10. Nourse J, et al. Chromosomal translocation $t(1 ; 19)$ results in synthesis of a homeobox fusion mRNA that codes for a potential chimeric transcription factor. Cell. 1990;60(4):535-545.

11. Borowitz MJ, et al. Predictability of the $t(1 ; 19)$ (q23;p13) from surface antigen phenotype: implications for screening cases of childhood acute lymphoblastic leukemia for molecular analysis: a Pediatric Oncology Group study. Blood. 1993;82(4):1086-1091.

12. Hardy RR, Kincade PW, Dorshkind K. The protean nature of cells in the B lymphocyte lineage. Immunity. 2007;26(6):703-714.

13. Geng $\mathrm{H}$, et al. Self-enforcing feedback activation between BCL6 and pre-B cell receptor signaling defines a distinct subtype of acute lymphoblastic leukemia. Cancer Cell. 2015;27(3):409-425.

14. Nahar R, et al. Pre-B cell receptor-mediated activation of BCL6 induces pre-B cell quiescence through transcriptional repression of MYC. Blood. 2011;118(15):4174-4178.

15. Irish JM, et al. B-cell signaling networks reveal a negative prognostic human lymphoma cell subset that emerges during tumor progression. Proc Natl Acad Sci U S A. 2010;107(29):12747-12754.

16. Horcher M, Souabni A, Busslinger M. Pax5/BSAP maintains the identity of B cells in late B lymphopoiesis. Immunity. 2001;14(6):779-790.
17. Mar BG, et al. Mutations in epigenetic regulators including SETD2 are gained during relapse in paediatric acute lymphoblastic leukaemia. Nat Commun. 2014;5:3469.

18. Bercovich D, et al. Mutations of JAK2 in acute lymphoblastic leukaemias associated with Down's syndrome. Lancet. 2008;372(9648):1484-1492.

19. Mullighan CG, et al. JAK mutations in high-risk childhood acute lymphoblastic leukemia. Proc Natl Acad Sci U S A. 2009;106(23):9414-9418.

20. Harvey RC, et al. Identification of novel cluster groups in pediatric high-risk B-precursor acute lymphoblastic leukemia with gene expression profiling: correlation with genome-wide DNA copy number alterations, clinical characteristics, and outcome. Blood. 2010;116(23):4874-4884.

21. Zhang J, et al. Key pathways are frequently mutated in high-risk childhood acute lymphoblastic leukemia: a report from the Children's Oncology Group. Blood. 2011;118(11):3080-3087.

22. Kawamura M, et al. Mutations of the p53 and ras genes in childhood $\mathrm{t}(1 ; 19)$-acute lymphoblastic leukemia. Blood.1995;85(9):2546-2552.

23. Harrison $\mathrm{C}$, et al. JAK inhibition with ruxolitinib versus best available therapy for myelofibrosis. N Engl J Med. 2012;366(9):787-798.

24. Verstovsek S, et al. A double-blind, placebo- 
controlled trial of ruxolitinib for myelofibrosis. N Engl J Med. 2012;366(9):799-807.

25. Quintás-Cardama A, et al. Preclinical characterization of the selective JAK1/2 inhibitor INCB018424: therapeutic implications for the treatment of myeloproliferative neoplasms. Blood. 2010;115(15):3109-3117.

26. Tefferi A. JAK inhibitors for myeloproliferative neoplasms: clarifying facts from myths. Blood. 2012;119(12):2721-2730.

27. Dedera DA, et al. Chimeric homeobox gene E2A-PBX1 induces proliferation, apoptosis, and malignant lymphomas in transgenic mice. Cell. 1993;74(5):833-843.

28. Monica K, LeBrun DP, Dedera DA, Brown R, Cleary ML. Transformation properties of the E2A-Pbx1 chimeric oncoprotein: fusion with E2A is essential, but the $\mathrm{Pbx} 1$ homeodomain is dispensable. Mol Cell Biol. 1994;14(12):8304-8314.

29. Kamps MP, Baltimore D. E2A-Pbx1, the t(1;19) translocation protein of human pre-B-cell acute lymphocytic leukemia, causes acute myeloid leukemia in mice. Mol Cell Biol.1993;13(1):351-357.

30. Thorsteinsdottir U, Krosl J, Kroon E, Haman A, Hoang T, Sauvageau G. The oncoprotein E2A-Pbx1a collaborates with Hoxa9 to acutely transform primary bone marrow cells. Mol Cell Biol. 1999;19(9):6355-6366.

31. Carroll AJ, Crist WM, Parmley RT, Roper M, Cooper MD, Finley WH. Pre-B cell leukemia associated with chromosome translocation 1;19. Blood. 1984;63(3):721-724.

32. Privitera E, et al. Different molecular consequences of the 1;19 chromosomal translocation in childhood B-cell precursor acute lymphoblastic leukemia. Blood.1992;79(7):1781-1788.

33. Grossmann V, et al. Whole-exome sequencing identifies somatic mutations of BCOR in acute myeloid leukemia with normal karyotype. Blood. 2011;118(23):6153-6163.

34. Liu GJ, et al. Pax 5 loss imposes a reversible differentiation block in B-progenitor acute lymphoblastic leukemia. Genes Dev. 2014;28(12):1337-1350.

35. Barber KE, et al. Molecular cytogenetic characterization of TCF3 (E2A)/19p13.3 rearrangements in $\mathrm{B}$-cell precursor acute lymphoblastic leukemia.
Genes Chromosomes Cancer. 2007;46(5):478-486.

36. Moorman AV, et al. Prognostic effect of chromosomal abnormalities in childhood B-cell precursor acute lymphoblastic leukaemia: results from the UK Medical Research Council ALL97/99 randomised trial. Lancet Oncol. 2010;11(5):429-438.

37. Hof J, et al. Mutations and deletions of the TP53 gene predict nonresponse to treatment and poor outcome in first relapse of childhood acute lymphoblastic leukemia. JClin Oncol. 2011;29(23):3185-3193.

38. Mullighan CG, et al. Rearrangement of CRLF2 in B-progenitor- and Down syndrome-associated acute lymphoblastic leukemia. Nat Genet. 2009;41(11):1243-1246.

39. Coustan-Smith E, et al. New markers for minimal residual disease detection in acute lymphoblastic leukemia. Blood. 2011;117(23):6267-6276.

40. Haferlach T, et al. Clinical utility of microarray-based gene expression profiling in the diagnosis and subclassification of leukemia: report from the International Microarray Innovations in Leukemia Study Group. J Clin Oncol. 2010;28(15):2529-2537.

41. Chan TO, et al. Small GTPases and tyrosine kinases coregulate a molecular switch in the phosphoinositide 3-kinase regulatory subunit. Cancer Cell. 2002;1(2):181-191.

42. Barretina J, et al. The Cancer Cell Line Encyclopedia enables predictive modelling of anticancer drug sensitivity. Nature. 2012;483(7391):603-607.

43. Pan L, Hanrahan J, Li J, Hale LP, Zhuang Y. An analysis of $\mathrm{T}$ cell intrinsic roles of $\mathrm{E} 2 \mathrm{~A}$ by conditional gene disruption in the thymus. J Immunol. 2002;168(8):3923-3932.

44. Rickert RC, Roes J, Rajewsky K. B lymphocyte-specific, Cre-mediated mutagenesis in mice. Nucleic Acids Res. 1997;25(6):1317-1318.

45. Kühn R, Schwenk F, Aguet M, Rajewsky K. Inducible gene targeting in mice. Science. 1995;269(5229):1427-1429.

46. Hobeika E, et al. Testing gene function early in the B cell lineage in mb1-cre mice. Proc Natl Acad Sci U S A. 2006;103(37):13789-13794.

47. Krutzik PO Nolan GP. Intracellular phospho-protein staining techniques for flow cytometry: mon- itoring single cell signaling events. Cytometry $A$. 2003;55(2):61-70.

48. Kuo HP, et al. Epigenetic roles of MLL oncoproteins are dependent on NF-кB. Cancer Cell. 2013;24(4):423-437.

49. Kotecha N, Krutzik PO, Irish JM. Web-based analysis and publication of flow cytometry experiments. Curr Protoc Cytom. 2010; Chapter 10:Unit 10.17.

50. Li H, et al. The Sequence alignment/map (SAM) format SAMtools. Bioinformatics. 2009;25(16):2078-2079.

51. McKenna A, et al. The Genome Analysis Toolkit: a MapReduce framework for analyzing nextgeneration DNA sequencing data. Genome Res. 2010;20(9):1297-1303.

52. Cingolani $\mathrm{P}$, et al. A program for annotating and predicting the effects of single nucleotide polymorphisms, SnpEff: SNPs in the genome of Drosophila melanogaster strain w1118; iso-2; iso-3. Fly (Austin). 2012;6(2):80-92.

53. McLaren W, Pritchard B, Rios D, Chen Y, Flicek $\mathrm{P}$, Cunningham F. Deriving the consequences of genomic variants with the Ensembl API and SNP Effect Predictor. Bioinformatics. 2010;26(16):2069-2070.

54. Thorvaldsdóttir H, Robinson JT, Mesirov JP. Integrative Genomics Viewer (IGV): highperformance genomics data visualization and exploration. Brief Bioinform. 2013;14(2):178-192.

55. Huang DW, Sherman BT, Lempicki RA. Systematic and integrative analysis of large gene lists using DAVID Bioinformatics Resources. Nature Protoc. 2009;4(1):44-57.

56. Huang DW, Sherman BT, Lempicki RA. Bioinformatics enrichment tools: paths toward the comprehensive functional analysis of large gene lists. Nucleic Acids Res. 2009;37(1):1-13.

57. Brochet X, Lefranc MP, Giudicelli V. IMGT/VQUEST: the highly customized and integrated system for IG and TR standardized V-J and V-D-J sequence analysis. Nucleic Acids Res. 2008;36(Web Server issue):W503-W508.

58. Schneider CA, Rasband WS, Eliceiri KW. NIH Image to ImageJ: 25 years of image analysis. Nat Methods. 2012;9(7):671-675. 